\title{
Was ist schwierig am Modellieren? Identifikation und Beschreibung von Hindernissen in Modellierungsprozessen von Lehramtsstudierenden naturwissenschaftlicher Fächer
}

\author{
Maximilian Göhner ${ }^{1}$ (D) $\cdot$ Moritz Krell ${ }^{1}$ \\ Eingegangen: 8. September 2020 / Angenommen: 24. Mai 2021 / Online publiziert: 28. Juni 2021 \\ (c) Der/die Autor(en) 2021
}

\section{Zusammenfassung}

Die Entwicklung von Modellkompetenz im naturwissenschaftlichen Unterricht kann potenziell zur Erreichung vielfältiger Ziele naturwissenschaftlicher Bildung beitragen. Studien deuten allerdings darauf hin, dass Modellierungsprozesse, in denen Modelle kritisch reflektiert oder als Werkzeuge zur Erkenntnisgewinnung eingesetzt werden, im Unterricht eher selten umgesetzt werden und dass Lehrkräfte in Bezug auf Fähigkeiten des naturwissenschaftlichen Modellierens weitere Förderung benötigen. Das Ziel der vorliegenden Studie ist die Identifikation und Beschreibung von Hindernissen, die in Modellierungsprozessen von Lehramtsstudierenden naturwissenschaftlicher Fächer auftreten. Hierzu wurden die Modellierungsprozesse von 36 Lehramtsstudierenden naturwissenschaftlicher Fächer bei der Untersuchung einer Blackbox qualitativ-inhaltsanalytisch ausgewertet. Es konnten 13 verschiedene Hinderniskategorien identifiziert und beschrieben werden. Die identifizierten Hinderniskategorien weisen teils Parallelen zum Experimentieren und naturwissenschaftlichen Arbeiten allgemein auf: Spezifische Hinderniskategorien für das Modellieren ergeben sich dagegen beim Umgang mit Analogien und Erfahrungen und treten vor allem beim Testen des entwickelten Modells auf. Basierend auf vorherigen Arbeiten wurden zudem die Modellierungsprozesse der Lehramtsstudierenden analysiert und sechs typischen Modellierungsstrategien zugeordnet. Es scheint kein eindeutiger Zusammenhang zwischen den identifizierten Hindernissen und den Modellierungsstrategien vorzuliegen, da ProbandInnen, die ähnlichen Hindernissen begegnen, sich hinsichtlich ihrer Modellierungsstrategien teils deutlich unterscheiden. Es wird diskutiert, inwiefern die identifizierten Hinderniskategorien für die weitere Entwicklung von Diagnoseinstrumenten und zur gezielten Planung von Förderangeboten genutzt werden können.

Schlüsselwörter Modellkompetenz $\cdot$ Naturwissenschaftliches Modellieren $\cdot$ Hindernisse $\cdot$ Lehramtsstudierende . Qualitative Inhaltsanalyse

Maximilian Göhner

maximilian.goehner@fu-berlin.de

1 Didaktik der Biologie, Freie Universität Berlin,

Schwendenerstraße 1, 14195 Berlin, Deutschland 


\title{
What Is Difficult in Modeling? the Identification and Description of Challenges Pre-service Science Teachers Encounter in Modeling Processes
}

\begin{abstract}
The development of modeling competence can potentially support the achievement of diverse goals in science education. However, studies suggest that modeling processes in which models are critically reflected or used as epistemic tools are rarely implemented in educational settings. Other studies propose a need to promote science teachers' abilities regarding scientific modeling. The present study aims to identify and describe challenges, which pre-service science teachers encounter while modeling. Therefore, the modeling practice of 36 pre-service science teachers engaging in a blackbox activity was analyzed using a qualitative content analytic approach. 13 different challenges were identified and described. Some of the identified challenges show parallels to previously identified challenges related to experimentation and scientific practices in general. In contrast, challenges, which seem to be specific for modeling are related to the use of analogies and occur mostly when the developed models are tested. Based on previous results, the pre-service science teachers' modeling practices were assigned to one of six types of modeling strategies. The identified challenges and the type of modeling strategy used by the pre-service science teachers seem not to be related, as pre-service science teachers encountering similar challenges can utilize very different modeling strategies. It is discussed, how the identified challenges can be used to support the development of diagnostic instruments as well as targeted interventions of modeling competence.
\end{abstract}

Keywords Modeling competence $\cdot$ Scientific modeling $\cdot$ Challenges $\cdot$ Pre-service science teachers $\cdot$ Qualitative content analysis

\section{Modellieren in den Naturwissenschaften}

Die Entwicklung von Kompetenzen naturwissenschaftlicher Erkenntnisgewinnung ist ein wichtiges Element der naturwissenschaftlichen Grundbildung (Bybee 2002; Osborne 2013), da diese Kompetenzen benötigt werden, um aktiv am naturwissenschafts- und technologieorientierten gesellschaftlichen Leben teilhaben zu können (Driver et al. 1996; Sharon und Baram-Tsabari 2020). Modellieren wird als zentrale Praxis der naturwissenschaftlichen Erkenntnisgewinnung betrachtet (Giere et al. 2006). Dabei wird der Prozess des naturwissenschaftlichen Modellierens zusammenfassend als Entwicklung und Evaluation von Repräsentationen natürlicher Systeme beschrieben (Godfrey-Smith 2006). Hierbei wird die Bezeichnung von Modellen als Repräsentationen allerdings auch kritisch diskutiert und alternativ epistemic tools (Knuuttila 2011) vorgeschlagen, um weniger den abbildenden und mehr den forschungsmethodischen Charakter von Modellen als Werkzeuge zur Erkenntnisgewinnung zu betonen (Gilbert und Justi 2016). Dem Verständnis von Modellen als Werkzeuge zur Erkenntnisgewinnung folgend, umfasst der Prozess des naturwissenschaftlichen Modellierens Untersuchungen in der empirischen Erfahrungswelt, die Entwicklung von Modellen als Erklärungen und Hypothesen in der theoretischen Modellwelt, sowie die Überprüfung dieser Erklärungen und Hypothesen durch weitere empirische Untersuchungen (DounasFrazer et al. 2018; Krell et al. 2016). Hierbei findet während des Modellierens ein Vergleich zwischen Modellwelt und Erfahrungswelt statt (Leisner-Bodenthin 2006), der den
Modellierenden im besten Fall bewusst ist und als solcher reflektiert wird (Mikelskis-Seifert und Leisner 2005).

Abb. 1 zeigt die dieser Studie zugrundeliegende Konzeptualisierung des Modellierens (Göhner und Krell 2018), welche auf der Basis naturwissenschaftstheoretischer und fachdidaktischer Arbeiten zum Modellieren entwickelt wurde (z.B. Clement 1989; Giere et al. 2006). Modellierungsprozesse werden darin als Sequenz von beobachtbaren Modellierungstätigkeiten aufgefasst, die wiederum drei Phasen des Modellierens zugeordnet werden können. Zur Phase der Exploration gehört die Beobachtung eines Phänomens in der Erfahrungswelt (Dounas-Frazer et al. 2018; Krell et al. 2016; Leisner-Bodenthin 2006; Mikelskis-Seifert und Leisner 2005). Es findet eine Exploration dieses Phänomens statt, welche sowohl unsystematisch als auch strukturiert und systematisch sein kann. Hierbei hat die Explorationsphase in der Regel das Ziel, Daten zu generieren und Regelmäßigkeiten oder Muster in diesen Daten zu entdecken. Eine Auswahl der in der Explorationsphase gewonnenen Daten und insbesondere deren Regelmäßigkeiten werden in die Modellwelt übertragen (Dounas-Frazer et al. 2018; Krell et al. 2016; Leisner-Bodenthin 2006; Mikelskis-Seifert und Leisner 2005). In der Herstellungsphase wird auf Grundlage von Analogien und Erfahrungen ein initiales Modell entwickelt, in dem die für das Phänomen als relevant erachteten Variablen des betrachteten Systems isoliert sind. Hierbei können zum Beispiel Analogien (,,[a] somewhat similar and familiar case in memory"; Clement 2008, S. 438) oder Erfahrungen, die eher mechanistischer Natur sind, zur Modellentwicklung herangezogen werden. Modelle können dabei vielfältige Repräsentationsformen aufweisen, zum Beispiel 


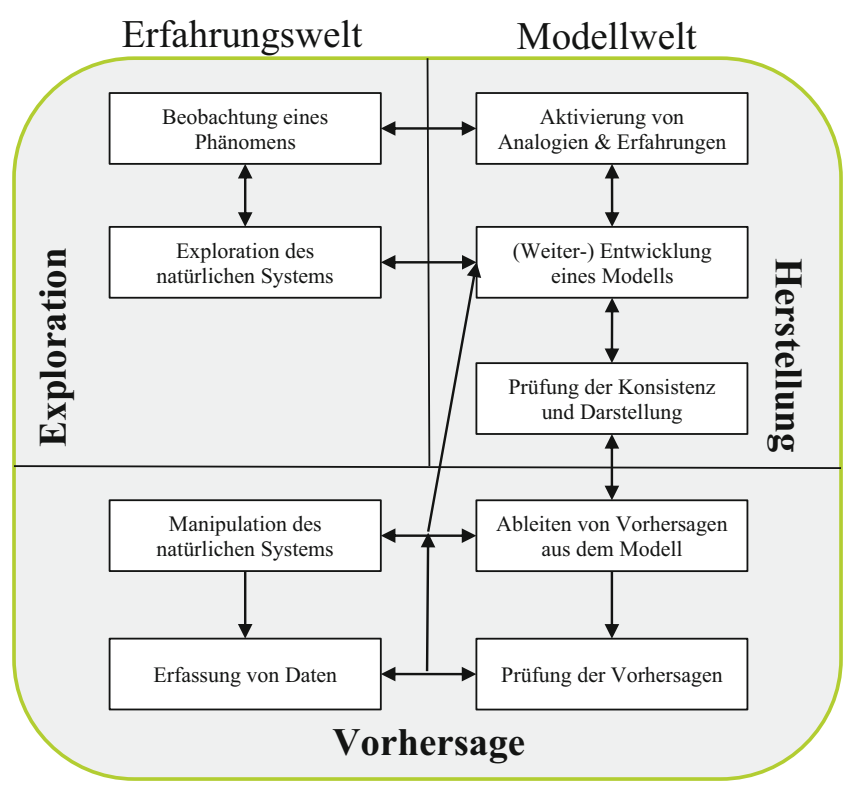

Abb. 1 Prozessdiagramm des Modellierens (Göhner und Krell 2018)

als Zeichnung (Chittleborough und Treagust 2007; Quillin und Thomas 2015) oder digital (Sins et al. 2005; Bo et al. 2018). Das entwickelte Modell kann dann auf interne Konsistenz sowie eine angemessene Darstellung des beobachteten Phänomens geprüft werden. Diese erkenntniserweiternde Entwicklung erklärender Modelle beruht auf abduktivem Schließen (Magnani 1999; Krüger und Upmeier zu Belzen 2021). Darüber hinaus sind auch deduktive Schlüsse möglich, indem in der Phase der Vorhersage eine gedankliche oder materielle Manipulation des Modells stattfindet, die es erlaubt Hypothesen darüber abzuleiten, wie sich das System unter bestimmten Bedingungen verhalten wird (Giere et al. 2006). Können die abgeleiteten Hypothesen falsifiziert werden, kann indirekt auf eine mangelnde Passung zwischen Modell und System geschlossen werden. Das Modell sollte dann überarbeitet oder gänzlich verworfen werden. In beiden Fällen schließt sich eine erneute Testung im beschriebenen Sinne an (Krell et al. 2016). Das Modellieren wird somit als zyklischer, nicht-linearer Prozess verstanden. Konkrete Modellierungsprozesse können dabei eine vielfältige, nicht notwendigerweise vollständige Abfolge beobachtbarer Tätigkeiten des Modellierens umfassen, die wie beschrieben den drei Phasen des Modellierens zugeordnet werden können.

\section{Modellkompetenz als Ziel naturwissenschaftlichen Unterrichts}

Modellkompetenz wird definiert als die Fähigkeiten, ,mit Modellen zweckbezogen Erkenntnisse gewinnen zu können und über Modelle mit Bezug auf ihren Zweck urteilen zu können, die Fähigkeiten, über den Prozess der Erkenntnisgewinnung durch Modelle und Modellierungen [...] zu reflektieren sowie die Bereitschaft, diese Fähigkeiten in problemhaltigen Situationen anzuwenden“(Upmeier zu Belzen und Krüger 2010). Insbesondere die Fähigkeiten, Modelle zur Vorhersage von Daten einzusetzen und sie als hypothetische Entitäten zu verstehen, werden als bedeutsame Elemente ausgeprägter Modellkompetenz betrachtet (Gouvea und Passmore 2017; Upmeier zu Belzen und Krüger 2010). In der Literatur werden darüber hinaus verschiedene Dimensionen von Modellkompetenz unterschieden; hierzu gehören 1) das Verständnis über Modelle und den Prozess des Modellierens (Modellverstehen bzw. metamodeling knowledge), 2) Fähigkeiten des praktischen Modellierens sowie, in einzelnen Quellen, ebenfalls 3) die Qualität des entwickelten Modells (Chiu und Lin 2019; Nicolaou und Constantinou 2014; Nielsen und Nielsen 2019).

Die Entwicklung von Modellkompetenz im naturwissenschaftlichen Unterricht kann potenziell zum Erreichen vielfältiger Ziele naturwissenschaftlicher Bildung beitragen (Hodson 2014; Justi und Gilbert 2002). So können naturwissenschaftliche Inhalte mit Hilfe von Modellen erschlossen werden, wenn Modelle als Produkte der Wissenschaft verstanden werden (Acher et al. 2007; Vo et al. 2019). Darüber hinaus kann naturwissenschaftliche Praxis reflektiert werden, wenn Modellierungsprozesse umgesetzt und nachvollzogen werden (Fleige et al. 2012). Aufgrund der groBen Bedeutung des Modellierens für die naturwissenschaftliche Erkenntnisgewinnung wird die Arbeit mit Modellen in Steuerungsdokumenten für den naturwissenschaftlichen (Schul-)Unterricht unterschiedlicher Länder explizit gefordert (ACARA 2015; BCMOE 2019; KMK 2005, 2020; NGSS Lead States 2013). In den Bildungsstandards im Fach Biologie für den Mittleren Schulabschluss in Deutschland wird hervorgehoben, dass ,gerade das Modellieren bzw. kritische Reflektieren des Modells bedeutsamer Teil der naturwissenschaftlichen Erkenntnisgewinnung" (KMK 2005, S. 11) ist. So sollen SchülerInnen am Ende der Jahrgangsstufe 10 zum Beispiel in der Lage sein, „Modelle praktisch [zu] erstellen“ (Anforderungsbereich (AFB) I), ,Sachverhalte mit Modellen [zu] erklären“ (AFB II) und „Hypothesen [zu] erstellen mit einem Modell“" (AFB III) (KMK 2005, S. 17).

Studien zum Einsatz von Modellen und dem Modellieren im Unterricht weisen allerdings darauf hin, dass diese curricularen Vorgaben oftmals nicht erreicht werden. So werden Modellierungsprozesse, in denen Modelle kritisch reflektiert oder als Werkzeuge zur Erkenntnisgewinnung eingesetzt werden, im Unterricht eher selten umgesetzt (Campbell et al. 2015). Im naturwissenschaftlichen Unterricht scheint das Entwickeln und Evaluieren von Modellen häufiger umgesetzt zu werden als die sich daran anschließende Weiterentwicklung von Modellen (Khan 2011). 
Insbesondere das Testen von Modellen durch die Ableitung und Überprüfung von Hypothesen scheint im Unterricht eher selten umgesetzt zu werden (Krell und Krüger 2013); obwohl gerade ein Verständnis dieses Testens von Modellen als ein Schlüssel zur Förderung von Modellverstehen betrachtet wird (Krell und Krüger 2016; Lee und Kim 2014). In der Videostudie von Werner et al. (2019) konnten Aufgabenstellungen, die Modelle als Werkzeuge zur Erkenntnisgewinnung nutzen, sowie die kritische Reflexion von Modellen nur selten beobachtet werden. Insgesamt kann somit davon ausgegangen werden, dass Modelle im Unterricht entgegen der curricularen Vorgaben (ACARA 2015; BCMOE 2019; KMK 2005, 2020; NGSS Lead States 2013) und fachdidaktischen Zielsetzungen (Fleige et al. 2012) primär als Anschauungsmedien zur Erschließung naturwissenschaftlicher Inhalte eingesetzt werden. Das Potenzial, das Modelle und das Modellieren dabei für die Unterrichtsgestaltung zur Förderung von Kompetenzen naturwissenschaftlicher Erkenntnisgewinnung mitbringen, wird somit offenbar eher selten genutzt.

Um den curricularen Vorgaben gerecht zu werden, sollen Lehrkräfte selbst über ausreichende Modellkompetenz verfügen (Günther et al. 2017, 2019), die sie befähigt im Unterricht eine Diagnose der Modellkompetenz von SchülerInnen, sowie die Planung und Umsetzung von entsprechenden Lerngelegenheiten vornehmen zu können (Elby und Hammer 2010; Sung und Oh 2018). Entsprechend wird auch im fachspezifischen Kompetenzprofil Biologie der ländergemeinsamen inhaltlichen Anforderungen in der Lehrerbildung gefordert, dass StudienabsolventInnen „,vertraut [sind] mit basalen Arbeits- und Erkenntnismethoden der Biologie und über Kenntnisse und Fertigkeiten sowohl im hypothesengeleiteten Experimentieren und Modellieren [,...] [verfügen]“ (KMK 2019). Diverse Studien weisen allerdings darauf hin, dass bei Lehrkräften in den verschiedenen Dimensionen der Modellkompetenz Defizite vorliegen (z.B. Krell und Krüger 2016; Nielsen und Nielsen 2019).

\section{Bisherige Forschungsergebnisse zur Modellkompetenz von Lehrkräften naturwissenschaftlicher Fächer}

Mehrere Studien deuten darauf hin, dass angehende (z.B. Torres und Vasconcelos 2015; Yenilmez Turkoglu und Oztekin 2016) sowie praktizierende (z.B. Justi und Gilbert 2003; Krell und Krüger 2016) Lehrkräfte über ein eher eingeschränktes Modellverstehen verfügen und Modelle vor allem als Repräsentationen oder direkte Abbilder der Realität betrachten. Ein Verständnis über Modelle als Werkzeuge zur naturwissenschaftlichen Erkenntnisgewinnung ist bei
Lehrkräften in der Regel nur gering ausgeprägt (Gilbert und Justi 2016).

Es liegen, anders als in anderen Bereichen der naturwissenschaftlichen Erkenntnisgewinnung (z.B. Experimentieren: Baur 2018; Hammann 2004), nur eingeschränkt qualitative und prozessorientierte Studien zu Fähigkeiten und Vorgehensweisen von Lehrkräften beim Modellieren vor (Louca und Zacharia 2012; Nicolaou und Constantinou 2014). Insbesondere liegen kaum Studien vor, die gezielt Interventionen zur Förderung des Verstehens von Modellierungsprozessen untersuchen (Campbell et al. 2015).

Die vorliegenden Studien zu Modellierungsprozessen von Lehrkräften beobachten, dass Lehrkräfte eher zu einer produkt-orientierten Perspektive auf Modelle und das Modellieren tendieren (Nielsen und Nielsen 2019) und dass Lehrkräfte selten Modellierungsprozesse umsetzen, in denen Modelle zur Vorhersage von Daten eingesetzt werden (Göhner und Krell 2020a). In der Studie von Göhner und Krell (2020a) zum Modellieren an einer Blackbox setzten lediglich fünf von 32 angehendenden Lehrkräften Modellierungsprozesse um, in denen wiederholt Vorhersagen aus entwickelten Modellen abgeleitet und überprüft wurden, während die Mehrzahl der ProbandInnen ausschließlich Tätigkeiten der Exploration und Herstellung zeigten (Abb. 1). Cheng et al. (2019) konnten zwar im Hinblick auf das Modellieren von Magnetismus beobachten, dass Lehrkräfte im Vergleich zu SchülerInnen elaboriertere Modelle entwickeln und eher wissenschaftliche Kriterien zur Evaluation der Modelle nutzen. Allerdings gelang dies auch nur etwa einem Drittel der dort teilnehmenden Lehrkräfte. Auch Windschitl und Thompson (2006) konnten in einer Interventionsstudie zeigen, dass es Lehrkräften schwerfällt, selbst Modelle zu entwickeln und als Werkzeuge zur Erkenntnisgewinnung zu nutzen. Zwar gelang es den Lehrkräften oftmals, über vorhandene wissenschaftliche Modelle elaboriert zu sprechen, beim selbstständigen Modellieren fiel es den teilnehmenden Lehrkräften allerdings schwer, theoretische Beziehungen in ihre Modelle zu integrieren, die ihnen nicht vollständig bekannt waren. Mehrere Studien beobachten zudem sogenanntes modelfitting Verhalten, bei dem der Fokus auf dem Output eines Modells liegt, anstatt auf den modellierten theoretischen Beziehungen; Modellparameter werden hierbei schrittweise angepasst, sodass der Output des Modells den tatsächlichen Beobachtungen entspricht, ohne tiefergehend über das Modell, die darin enthaltenen Elemente oder ihre Beziehungen zueinander nachzudenken (Hogan und Thomas 2001; Sins et al. 2005).

Neben einem mangelnden Modellverstehen wird auch mangelndes Fachwissen als ein Faktor vermutet, der Modellierungsprozesse erschwert (Ruppert et al. 2019). Demgegenüber beobachten Windschitl und Thompson (2006), dass es Lehrkräften oft nicht gelingt, richtig erkannte $\mathrm{Zu}$ - 
sammenhänge und Konzepte über ein beobachtetes Phänomen in Modellierungsprozessen zu berücksichtigen und in die entwickelten Modelle zu integrieren. Selbst Lehrkräfte mit ausreichendem Fachwissen identifizieren zwar relevante Konzepte, aber entwickeln oft kein Modell, welches das vorhandene Wissen über das gegebene Phänomen akkurat repräsentiert. Diese unvollständigen Modelle wiederum behindern den weiteren Prozess des Modellierens, da sie nicht sinnvoll im Zusammenhang mit dem gegebenen Phänomen getestet werden können (Windschitl und Thompson 2006).

Es liegen keine Studien zur Entwicklung von Modellkompetenz bei angehenden Lehrkräften über den Studienverlauf vor, die systematisch untersuchen, inwieweit die curricularen Forderungen in Bezug auf das Modellieren (KMK 2019) in der universitären Lehkräftebildung erreicht werden. Es ist demnach auch möglich, dass Lehrkräfte oftmals wenige Gelegenheiten haben, an Prozessen der naturwissenschaftlichen Erkenntnisgewinnung durch Modellieren teilzuhaben und diese explizit zu reflektieren. Studien zur Entwicklung von Kompetenzen wissenschaftlichen Denkens (inkl. der Dimension Modellieren) zeigen eine positive aber lediglich moderate Entwicklung dieser Kompetenzen bei Lehramtsstudierenden naturwissenschaftlicher Fächer im Verlauf des Lehramtsstudiums (Krüger et al. 2020). Hartmann et al. (2015) schlussfolgern, dass für die Entwicklung von Kompetenzen naturwissenschaftlicher Erkenntnisgewinnung eine gezielte und explizite Förderung und Reflexion über die Prozesse naturwissenschaftlicher Erkenntnisgewinnung notwendig ist.

Zusammenfassend kann davon ausgegangen werden, dass die Förderung von Modellverstehen und Fachwissen nicht ausreicht, um bei Lehrkräften Fähigkeiten des naturwissenschaftlichen Modellierens zu entwickeln, sodass diese in der Lage sind, Modellierungsprozesse erfolgreich in die Unterrichtspraxis zu übertragen (Bo et al. 2018). Das bloße Nachahmen und Teilhaben an wissenschaftlichen Erkenntnisprozessen, beispielsweise während eines Lehramtsstudiums, reichen nicht zum Beherrschen ebenjener Prozesse aus (Bell et al. 2003). Eine gezielte und explizite Förderung ist nötig, um Modelle als Werkzeuge zur Erkenntnisgewinnung entwickeln und die Praxis des wissenschaftlichen Modellierens auch im schulischen Kontext umsetzen zu können (Clement 2000; Hartmann et al. 2015; Lehrer und Schauble 2006). Sung und Oh (2018) schlagen die Identifikation und Beschreibung von spezifischen Hindernissen vor, die in Modellierungsprozessen auftreten können. Naturwissenschaftliches Modellieren wird hierbei als Problemlösen verstanden (Mayer 2007). Ein Problem ist definiert durch das Vorhandensein eines Ziels, dessen Erreichen fraglich ist, und das Fehlen einer Routine, um Hindernisse auf dem Weg zu diesem Ziel zu beseitigen (Funke 2011; Mayer 2007). Ein Hindernis kann dabei verstanden werden als eine ,Stelle im Bearbeitungsprozess, in der rekonstruierbar ist, dass eine Person nichts oder etwas nicht selbstverständlich (im Sinne von nicht sicher, zweifelnd) ausführt und dabei auf nichts in der Aufgabensituation Anwendbares zugreifen möchte bzw. zugreifen kann“ (Lange, 2013, S. 32). Problemlösen bedeutet demnach ,das Beseitigen eines Hindernisses oder das Schließen einer Lücke in einem Handlungsplan durch bewusste kognitive Aktivitäten, die das Erreichen eines beabsichtigten Ziels möglich machen sollen“ (Funke 2011, S. 138). Die Identifikation spezifischer Hindernisse in Modellierungsprozessen könnte daher sowohl zur problemtypenbasierten Modellierung und Diagnose von Modellkompetenz (Gut et al. 2014) als auch in Vermittlungssituationen genutzt werden, in denen hindernisspezifisch Hilfestellungen gegeben und die Entwicklung von Modellkompetenz gezielt unterstützt werden könnte (Baur 2018; Sins et al. 2005; Sung und Oh 2018).

\section{Ziel und Fragestellungen}

Die vorliegende Studie hat das Ziel Hindernisse zu identifizieren und zu beschreiben, die in Modellierungsprozessen von Lehramtsstudierenden auftreten, sowie die identifizierten Hindernisse in den Modellierungsprozessen zu verorten. Hierbei werden drei Forschungsfragen verfolgt:

1. Welche Hindernisse lassen sich in den Modellierungsprozessen von Lehramtsstudierenden naturwissenschaftlicher Fächer identifizieren und beschreiben?

2. Inwiefern treten die identifizierten Hindernisse in einzelnen Phasen der Modellierungsprozesse von Lehramtsstudierenden naturwissenschaftlicher Fächer besonders häufig auf?

3. Inwiefern besteht ein Zusammenhang zwischen den identifizierten Hindernissen und den Modellierungsprozessen von Lehramtsstudierenden naturwissenschaftlicher Fächer?

\section{Methoden}

\section{Studienkontext}

Die vorliegende Studie ist im universitären Lehramtsstudium und damit in der ersten Phase der Lehrkräftebildung verortet. Lehrkräfte sollen bereits im Studium Teile des professionellen Wissens und der Kompetenzen entwickeln, die später im schulischen Alltag benötigt werden (Neumann et al. 2017); hierzu gehören auch Wissen und Kompetenzen im Bereich der Erkenntnisgewinnung (KMK 2019). 


\section{Setting der Studie}

Um Modellierungsprozesse anzuregen, wurde in der vorliegenden Studie ein Blackbox-Setting eingesetzt. Der Einsatz einer Blackbox stellt in der naturwissenschaftsdidaktischen Forschung einen etablierten Ansatz zur Untersuchung von Prozessen der Erkenntnisgewinnung und des wissenschaftlichen Denkens dar (Krell und Hergert 2019; Lederman und Abd-El-Khalick 2002; Passmore und Svoboda 2012). Die Blackbox repräsentiert hierbei ein natürliches Phänomen und die Untersuchung der Blackbox den Prozess der Erkenntnisgewinnung (Upmeier zu Belzen 2014). In der vorliegenden Studie wurde eine Wasser-Blackbox eingesetzt. Diese kann durch das Einfüllen von Wasser und die anschließende Beobachtung des resultierenden Outputs untersucht werden (Krell und Hergert 2019). Eine Darstellung des inneren Mechanismus der verwendeten Blackbox ist im Anhang enthalten (Abb. 6).

Die ProbandInnen wurden in einem Einführungsgespräch über das Ziel der Studie informiert, um ihr schriftliches Einverständnis zur Studienteilnahme gebeten und aufgefordert, einen kurzen Fragebogen zum Modellverstehen zu beantworten (Krell und Krüger 2016). Die nachfolgende Aufgabe der Untersuchung der Blackbox wurde dabei explizit als Modellierungsaufgabe angekündigt. Die ProbandInnen wurden anschließend aufgefordert, während der Untersuchung der Blackbox laut zu denken (Ericsson und Simon 1998), was ebenfalls im Rahmen des Einführungsgesprächs mit drei kurzen Aufgaben geübt wurde (Sandmann 2014). Im Anschluss an diese Vorbereitung wurden die ProbandInnen in einen Raum geführt, in welchem die Blackbox, Messbecher unterschiedlichen Fassungsvermögens, ein Wasserreservoir, eine Tafel mit Kreide und drei Kameras zur Dokumentation aufgestellt waren. Nach einer kurzen, standardisierten Erklärung der grundlegenden Funktionsweise der Blackbox wurde den ProbandInnen die folgende Aufgabenstellung verbal mitgeteilt: „Deine Aufgabe ist es, mit Hilfe der bereitgestellten Materialien das Verhalten der Blackbox zu untersuchen und ein Modell des Inneren der Blackbox zu entwickeln und fortlaufend auf einer Tafel zu dokumentieren. Die einzige Einschränkung ist: Du darfst die Blackbox nicht öffnen.“ Zudem war die Aufgabenstellung in Kurzform („Zeichnen Sie ein Modell des Inneren der Blackbox.") zu jeder Zeit als sichtbarer Hinweis in Form eines laminierten Schildes an der Tafel angebracht. Alle ProbandInnen wurden zudem darüber informiert, dass keine zeitliche Beschränkung vorliegt (durchschnittliche Untersuchungszeit: $1 \mathrm{~h} 08 \mathrm{~min}$; Anhang, Tab. 5). Während dieser Zeit verblieb der Erstautor oder eine geschulte studentische Hilfskraft im Raum, um die Technik zu überwachen und, falls nötig, die ProbandInnen mit standardisierten Impulsen dazu aufzufordern, weiterhin laut zu denken.

\section{Sampling}

In der vorliegenden Studie wurde ein Sampling nach einem qualitativen Stichprobenplan mit zwei Samplingkriterien umgesetzt (Schreier 2010). Das erste Kriterium sind Kompetenzen des wissenschaftlichen Denkens (scientific reasoning competencies; Hartmann et al. 2015), da wissenschaftliches Denken und wissenschaftliches Modellieren nach theoretischen Überlegungen positiv zusammenhängen (Giere et al. 2006; Passmore et al. 2014). Als zweites Kriterium wurden allgemeine kognitive Fähigkeiten ausgewählt, welche neben verbalen und numerischen Fähigkeiten auch Fähigkeiten des räumlichen Denkens umfassen, welche elementare Voraussetzung für Modellierungsprozesse sind (Nersessian 2002). Zwischen allgemeinen kognitiven Fähigkeiten und Modellverstehen (Krell et al. 2014) sowie wissenschaftlichem Problemlösen (Nehring et al. 2015) konnte auch in empirischen Studien ein positiver Zusammenhang gezeigt werden. Die beiden Kriterien wurden mit zwei etablierten paper-pencil Instrumenten (Hartmann et al. 2015; Liepmann et al. 2007) bei $N=666$ Lehramtsstudierenden naturwissenschaftlicher Fächer erhoben. Um die Wahrscheinlichkeit zu erhöhen, möglichst vielfältige Modellierungsprozesse beobachten zu können, wurden ausgehend von der jeweiligen Referenzstichprobe diejenigen Studierenden zum Hauptteil der vorliegenden Studie eingeladen, die für beide Kriterien eine (wissenschaftliches Denken) beziehungsweise eine halbe (kognitive Fähigkeiten) Standardabweichung besser oder schlechter abgeschnitten haben (heterogene Stichprobe; Schreier 2010). Dies traf auf 57 Studierende zu, von denen 36 letztlich teilnahmen (Anhang, Tab. 5). Eine Analyse der Modellierungsprozesse der ProbandInnen in Abhängigkeit der beiden Samplingkriterien ist andernorts publiziert (Göhner und Krell in Druck) und daher nicht Gegenstand der vorliegenden Arbeit.

\section{Analyse der Modellierungsprozesse}

Die Tonspuren der Videos wurden vollständig transkribiert, dabei wurden neben den Verbalisierungen der ProbandInnen auch ausgewählte Informationen zu ihrem Verhalten im Transkript ergänzt (z.B. Einfüllen von Wasser, Zeichnen eines Modells an der Tafel). Die daraus resultierenden Transkripte wurden nach einer strukturierenden qualitativen Inhaltsanalyse und unter Zuhilfenahme der Videos untersucht (Schreier 2012). Innerhalb der Modellierungsprozesse der ProbandInnen wurden hierbei einzelne Tätigkeiten des Modellierens identifiziert, wofür auf ein Kategoriensystem mit 19 Kategorien (=Tätigkeiten) zurückgegriffen wurde (Tab. 1). Die Identifikation der einzelnen Tätigkeiten in den Modellierungsprozessen der ProbandInnen wurde von zwei Personen unabhängig voneinander vorgenommen (Göhner und Krell 2020b). Dabei wurde im Mittel eine 
Tab. 1 Kategoriensystem mit 19 Tätigkeiten des Modellierens (Krell et al. 2019) sowie deren Verortung in Phasen des Modellierungsprozesses

\begin{tabular}{|c|c|c|c|}
\hline Phase & Oberkategorie & Kategorie (=Tätigkeit) & Beschreibung \\
\hline $\mathrm{E}$ & $\begin{array}{l}\text { Beobachtung eines } \\
\text { Phänomens }\end{array}$ & $\begin{array}{l}\text { 1. Beobachtung eines } \\
\text { Phänomens }\end{array}$ & $\begin{array}{l}\text { ProbandInnen nehmen Verhalten von Blackbox als spontan nicht erklärbar } \\
\text { wahr oder erkennen fehlende Passung zwischen angenommenem Muster } \\
\text { der Blackbox und Verhalten von Blackbox }\end{array}$ \\
\hline \multirow[t]{4}{*}{$\mathrm{E}$} & \multirow[t]{4}{*}{$\begin{array}{l}\text { Exploration des } \\
\text { Systems }\end{array}$} & $\begin{array}{l}\text { 2. Input/Output nicht } \\
\text { hypothesengeleitet }\end{array}$ & $\begin{array}{l}\text { ProbandInnen nehmen Eingabe von Input vor und/oder beobachten den } \\
\text { Output (explorativ; nicht hypothesengeleitet) }\end{array}$ \\
\hline & & 3. Zusammenfassung & $\begin{array}{l}\text { ProbandInnen fassen das beobachtete Verhalten der Blackbox zusammen } \\
\text { oder beschreiben es }\end{array}$ \\
\hline & & $\begin{array}{l}\text { 4. Input/Output hypo- } \\
\text { thesengeleitet Daten }\end{array}$ & $\begin{array}{l}\text { ProbandInnen nehmen Eingabe von Input vor und/oder beobachten den } \\
\text { Output (Mustererkennung) }\end{array}$ \\
\hline & & 5. Mustererkennung & ProbandInnen bestätigen/erkennen Muster \\
\hline E.H,V ${ }^{a}$ & $\begin{array}{l}\text { Aktivierung von Ana- } \\
\text { logien und Erfahrun- } \\
\text { gen }\end{array}$ & $\begin{array}{l}\text { 6. Analogien und Erfah- } \\
\text { rungen }\end{array}$ & $\begin{array}{l}\text { ProbandInnen formulieren Ideen über Mechanismus der Blackbox und/ } \\
\text { oder reflektieren Vor- und Nachteile ihrer Ideen und verwerfen ggf. Ideen } \\
\text { (ohne zeichnerische Umsetzung) }\end{array}$ \\
\hline \multirow[t]{4}{*}{$\mathrm{H}$} & \multirow[t]{4}{*}{$\begin{array}{l}\text { (Weiter-) Entwicklung } \\
\text { eines Modells }\end{array}$} & 7. Modellentwicklung & $\begin{array}{l}\text { ProbandInnen entwickeln auf Grundlage von Beobachtung, Analogien und/ } \\
\text { oder Erfahrungen zeichnerisch ein Modell der Blackbox }\end{array}$ \\
\hline & & $\begin{array}{l}\text { 8. Optimierung Modell- } \\
\text { objekt }\end{array}$ & $\begin{array}{l}\text { ProbandInnen entwickeln Modell weiter zur Optimierung von Funktionsfä- } \\
\text { higkeit, Ästhetik o. ä. (Modellobjekt) }\end{array}$ \\
\hline & & $\begin{array}{l}\text { 9. Optimierung Modell } \\
\text { retrospektiv }\end{array}$ & $\begin{array}{l}\text { ProbandInnen entwickeln Modell weiter aufgrund fehlerhafter Überein- } \\
\text { stimmungen mit Beobachtungen an Blackbox (retrospektiv) }\end{array}$ \\
\hline & & 10. Verwerfen Modell & $\begin{array}{l}\text { ProbandInnen verwerfen Modell aufgrund fehlerhafter Übereinstimmungen } \\
\text { mit Beobachtungen an Blackbox (retrospektiv; vgl. Kategorie 12) oder } \\
\text { mangelnder Konsistenz des Modellobjekts (vgl. Kategorie 11) }\end{array}$ \\
\hline \multirow[t]{2}{*}{$\mathrm{H}$} & \multirow{2}{*}{$\begin{array}{l}\text { Prüfung von } \\
\text { Konsistenz und } \\
\text { Darstellung }\end{array}$} & $\begin{array}{l}\text { 11. Prüfung Konsistenz } \\
\text { Modellobjekt }\end{array}$ & $\begin{array}{l}\text { ProbandInnen reflektieren/überprüfen/bewerten Konsistenz des Modells } \\
\text { (Modellobjekt) }\end{array}$ \\
\hline & & $\begin{array}{l}\text { 12. Prüfung Konsistenz } \\
\text { Modell/Daten }\end{array}$ & $\begin{array}{l}\text { ProbandInnen vergleichen Eigenschaften des Modells mit Beobachtungen } \\
\text { an Blackbox (retrospektiv) }\end{array}$ \\
\hline $\mathrm{H}$ & $\begin{array}{l}\text { Feststellung Konsis- } \\
\text { tenz \& Darstellung des } \\
\text { Modells }\end{array}$ & $\begin{array}{l}\text { 13. Feststellen Konsis- } \\
\text { tenz }\end{array}$ & ProbandInnen stellen Konsistenz und Angemessenheit des Modells fest \\
\hline $\mathrm{V}$ & $\begin{array}{l}\text { Ableiten von Vorher- } \\
\text { sage (Hypothese) aus } \\
\text { Modell }\end{array}$ & $\begin{array}{l}\text { 14. Hypothese aus Mo- } \\
\text { dell }\end{array}$ & $\begin{array}{l}\text { ProbandInnen nutzen Modell, um Vorhersage über Output bei bestimmtem } \\
\text { Input zu treffen }\end{array}$ \\
\hline \multirow[t]{3}{*}{$\mathrm{V}$} & \multirow{3}{*}{$\begin{array}{l}\text { Prüfung der } \\
\text { Vorhersage } \\
\text { (datenbasiert) }\end{array}$} & $\begin{array}{l}\text { 15. Input/Output hypo- } \\
\text { thesengeleitet Modell }\end{array}$ & $\begin{array}{l}\text { ProbandInnen nehmen Eingabe von Input vor und beobachten den Output } \\
\text { (hypothesengeleitet aus Modell) }\end{array}$ \\
\hline & & 16. Hypothese bestätigt & $\begin{array}{l}\text { ProbandInnen bestätigen Hypothese aus Modell durch Output von Black- } \\
\text { box }\end{array}$ \\
\hline & & $\begin{array}{l}\text { 17. Hypothese falsifi- } \\
\text { ziert }\end{array}$ & $\begin{array}{l}\text { ProbandInnen falsifizieren Hypothese aus Modell durch Output von Black- } \\
\text { box }\end{array}$ \\
\hline \multirow[t]{2}{*}{$\mathrm{V}$} & \multirow[t]{2}{*}{$\begin{array}{l}\text { Ändern/Verwerfen des } \\
\text { Modells (datenbasiert) }\end{array}$} & $\begin{array}{l}\text { 18. Optimierung Mo- } \\
\text { dell nach falsifizierter } \\
\text { Hypothese }\end{array}$ & $\begin{array}{l}\text { ProbandInnen weiterentwickeln Modell aufgrund von falsifizierter Hypo- } \\
\text { these (datenbasiert) }\end{array}$ \\
\hline & & $\begin{array}{l}\text { 19. Verwerfen Modell } \\
\text { nach falsifizierter Hypo- } \\
\text { these }\end{array}$ & $\begin{array}{l}\text { ProbandInnen verwerfen Modell aufgrund von falsifizierter Hypothese } \\
\text { (datenbasiert) }\end{array}$ \\
\hline
\end{tabular}

$E$ Exploration; $H$ Herstellung; $V$ Vorhersage (vgl. Abb. 1)

${ }^{a}$ Analogien und Erfahrungen können während des gesamten Modellierungsprozesses aktiviert werden. Diese Kategorie wird daher keiner spezifischen Phase zugeordnet

hohe Interrater-Übereinstimmung erreicht $(0,45 \leq \kappa \leq 0,93$; Mittelwert $_{\mathrm{K}}=0,77$; Landis und Koch 1977).

Auf der Grundlage der Komplexität und Homogenität der Tätigkeitsverläufe, das heißt dem Auftreten und der Abfolge der verschiedenen Tätigkeiten in den Modellierungsprozessen, wurden die ProbandInnen einer von sechs typischen Modellierungsstrategien zugeordnet (Tab. 2).
Diese Zuordnung der ProbandInnen basierend auf den konkret beobachteten Modellierungsprozessen $\mathrm{zu}$ typischen, idealisierten Modellierungsstrategien setzt keine aktiv von den ProbandInnen umgesetzte metakognitive Handlung voraus. Modellierungsprozesse werden als komplexer verstanden, umso mehr verschiedene Tätigkeiten des Modellierens sie umfassen (Göhner und Krell 2020a). Modellierungspro- 
Tab. 2 Typen von Modellierungsstrategien, deren Komplexität und Homogenität sowie die Häufigkeit des Auftretens in dieser Studie (Göhner und Krell 2020a)

\begin{tabular}{|c|c|c|c|c|}
\hline $\begin{array}{l}\text { Typ von Modellie- } \\
\text { rungsstrategie }\end{array}$ & Kurzbeschreibung & Häufigkeit & Komplexität & $\begin{array}{l}\text { Homo- } \\
\text { genität }\end{array}$ \\
\hline 1 Nur Exploration & $\begin{array}{l}\text { Es werden ausschließlich Tätigkeiten der Exploration (1-5) durchgeführt. Eine } \\
\text { Vermutung über den inneren Mechanismus der Blackbox wird lediglich verbal } \\
\text { kommuniziert, es entsteht kein gezeichnetes Modell }\end{array}$ & 1 & Niedrig & Niedrig \\
\hline $\begin{array}{l}\text { 2a Exploration } \\
\text { \& Herstellung; } \\
\text { Fokus Exploration }\end{array}$ & $\begin{array}{l}\text { Es werden Tätigkeiten der Exploration (1-5) und der Herstellung (7-13) durch- } \\
\text { geführt. Dabei überwiegen a Tätigkeiten der Exploration. Das gezeichnete Mo- } \\
\text { dell entsteht oft erst am Ende der Modellierungsprozesse und dient primär der } \\
\text { abschließenden Repräsentation der Vermutungen }\end{array}$ & 12 & Niedrig & Mittel \\
\hline $\begin{array}{l}\text { 2b Exploration } \\
\text { \& Herstellung; } \\
\text { Fokus Herstellung }\end{array}$ & $\begin{array}{l}\text { Es werden Tätigkeiten der Exploration (1-5) und der Herstellung (7-13) durch- } \\
\text { geführt. Dabei überwiegen }{ }^{\text {a }} \text { Tätigkeiten der Herstellung. Ein gezeichnetes Mo- } \\
\text { dell entsteht oft früh in Modellierungsprozessen und dient primär der fortlaufen- } \\
\text { den Entwicklung der Vermutungen }\end{array}$ & 2 & Mittel & Mittel \\
\hline $\begin{array}{l}\text { 2c Exploration \& } \\
\text { Herstellung }\end{array}$ & $\begin{array}{l}\text { Es werden Tätigkeiten der Exploration (1-5) und der Herstellung (7-13) durch- } \\
\text { geführt, ohne dass ein Schwerpunkt auf einer der beiden Phasen erkennbar ist. } \\
\text { Ein gezeichnetes Modell entsteht oft früh in Modellierungsprozessen und dient } \\
\text { primär der retrospektiven Kontrastierung der entwickelten Vermutung und der } \\
\text { beobachteten Daten }\end{array}$ & 6 & Mittel & Hoch \\
\hline $\begin{array}{l}\text { 3a Exploration, } \\
\text { Herstellung \& } \\
\text { Vorhersage }\end{array}$ & $\begin{array}{l}\text { Es werden Tätigkeiten der Exploration (1-5), der Herstellung (7-13) und der } \\
\text { Vorhersage (14-19) durchgeführt. Das gezeichnete Modell wird dabei einmalig } \\
\text { zur Vorhersage von nachfolgend generierten Daten eingesetzt }\end{array}$ & 9 & Hoch & Mittel \\
\hline $\begin{array}{l}\text { 3b Exploration, } \\
\text { Herstellung \& } \\
\text { mehrmalige Vor- } \\
\text { hersage }\end{array}$ & $\begin{array}{l}\text { Es werden Tätigkeiten der Exploration (1-5), der Herstellung (7-13) und der } \\
\text { Vorhersage (14-19) durchgeführt. Das gezeichnete Modell wird dabei mehrmals } \\
\text { zur Vorhersage von nachfolgend generierten Daten eingesetzt }\end{array}$ & 6 & Hoch & Hoch \\
\hline
\end{tabular}

${ }^{a}$ Das überwiegende Vorkommen von Tätigkeiten setzt voraus, dass mindestens $50 \%$ der Tätigkeiten in einem Modellierungsprozess der entsprechenden Phasen zugeordnet werden können

zesse werden als homogener verstanden, umso gleichmäBiger die Tätigkeiten des Modellierens vorkommen (d.h. alle Tätigkeiten werden ähnlich häufig ausgeführt und auch die Wechsel zwischen Tätigkeiten sind gleichmäßig verteilt). Die Komplexität und die Homogenität der Modellierungsprozesse wurden mit Hilfe graphentheoretischer Maße quantifiziert (Göhner und Krell 2020a). In Bezug auf die Qualität der Modellierungsprozesse, erfüllen nur Modellierungsprozesse, die Modellierungsstrategie Typ 3a oder $3 \mathrm{~b}$ zugeordnet werden, das curricular beschriebene Ideal der Modellierung zur naturwissenschaftlichen Erkenntnisgewinnung (ACARA 2015; BCMOE 2019; KMK 2005, 2020; NGSS Lead States 2013) und die Nutzung von Modellen zur Vorhersage von Daten (Gouvea und Passmore 2017; Upmeier zu Belzen und Krüger 2010). Diese beiden Modellierungsstrategien zeichnen sich durch das zyklische Durchlaufen vielfältiger Tätigkeiten des Modellierens aus, wobei wiederholt Daten gesammelt, Modelle entwickelt und anhand von Vorhersagen getestet werden.

\section{Identifikation der Hindernisse}

Um Hindernisse in den Modellierungsprozessen der ProbandInnen zu identifizieren wurden alle 36 Transkripte im Rahmen einer weiteren strukturierenden qualitativen Inhaltsanalyse analysiert. Hierbei wurde initial eine induktive
Strategie der Kategorienbildung verfolgt, da bisher wenige Studien zu Modellierungsprozessen von Lehrkräften vorliegen (Louca und Zacharia 2012; Nicolaou und Constantinou 2014). Zur Pilotierung des Kategoriensystems wurden hierbei insgesamt neun der 36 Fälle ausgewählt, die sich hinsichtlich ihres Vorgehens und der verwendeten Modellierungsstrategie unterscheiden. Die Transkripte wurden event-basiert kodiert. Hindernisse sind hierbei nach Dörner (1976) operationalisiert als Stellen im Modellierungsprozess an denen die ProbandInnen innehalten und äußern, dass sie die Aufgabe als schwierig empfinden oder ratlos sind, sowie Stellen, an denen die ProbandInnen längere Pausen beim lauten Denken machen und dann mit einer anderen Aktivität fortfahren (vgl. „,subjektive Barriere“; Dörner 1976). Zusätzlich wurden neben diesen Verbalisierungen, in denen die ProbandInnen von sich aus auf Hindernisse hinweisen oder längere Pausen machen, auch solche Textstellen markiert, in denen Verbalisierungen nicht der aktuell oder einer später ausgeführten Handlung entsprechen (vgl. „intersubjektive Barriere“; Dörner 1976). Basierend auf den markierten Textstellen von sechs der neun Transkripte wurden dann induktiv Kategorien von Hindernissen gebildet. Inhaltlich bestehen die Kodiereinheiten aus mindestens ganzen Sätzen, in denen entweder das Wahrnehmen eines Hindernisses verbalisiert wird, oder in denen Verbalisierungen enthalten sind, die sich später im Abgleich mit 
den Handlungen der ProbandInnen als unstimmig erkennen lassen. Zeitlich sind die Kodiereinheiten damit auf wenige Sekunden beschränkt, die zusätzlich in die Tätigkeiten des Modellierens als Kontexteinheit (wenige Sekunden bis mehrere Minuten) eingebettet sind. Der Erstautor und eine geschulte Mitarbeiterin haben in fünf Entwicklungszyklen abwechselnd Textstellen kategorisiert oder gegebenenfalls einzelne Kategorien weiter ausdifferenziert. Um das Kategoriensystem auch deduktiv zu verorten und Vergleichbarkeit sicherzustellen, wurden die zunächst induktiv gebildeten Kategorien mit vergleichbaren Studien, auch zu anderen naturwissenschaftlichen Arbeitsweisen und anderen Zielgruppen abgeglichen (Baur 2018; Hammann 2004; Hogan und Thomas 2001; Meister et al. 2020; Woolley et al. 2018). Dieser Entwicklungsprozess wurde begleitet durch die wiederholte Feststellung der Interrater-Übereinstimmung. Die Werte der Interrater-Übereinstimmung wurden dabei als Leitkriterium für das intersubjektive Verständnis über die Kategorien und deren Trennschärfe genutzt und bildeten die Basis für die Diskussion des Kategoriensystems und die Konsensfindung bei Nichtübereinstimmungen (Göhner und Krell 2020b). Die verbliebenen drei der neun zur Pilotierung des Kategoriensystems genutzten Fälle wurden vom Erstautor und der Mitarbeiterin analysiert, ohne weitere Änderungen am Kategoriensystem vorzunehmen. Die erneute Überprüfung der Interrater-Übereinstimmung über die drei Fälle hinweg zeigte mit $\kappa=0,66$ eine substantielle Übereinstimmung (Landis und Koch 1977). Das so pilotierte Kategoriensystem wurde dann auf die weiteren 27 Fälle angewendet, wobei sich weitere kleine Anpassungen am Kategoriensystem ergaben (Schreier 2012).

\section{Auswertung der Daten in Bezug auf die Fragestellungen}

Zur Beantwortung von Forschungsfragen 1 und 2 wurden die kodierten Hindernisse der ProbandInnen absolut und aufgeteilt nach den einzelnen Tätigkeiten und Phasen des Modellierens (Tab. 1) betrachtet. Zur Beantwortung von Forschungsfrage 3 wurde die Häufigkeit der einzelnen Hindernisse $z$-standardisiert und es wurde geprüft, inwiefern sich das Auftreten der identifizierten Hindernisse in Abhängigkeit der jeweils umgesetzten Modellierungsstrategie (Tab. 2) unterscheidet (ANOVA). Ergänzend wurde dies zwischen ProbandInnen, die Modelle zur Vorhersage genutzt haben (Strategien 3a, 3b; $n=15$ ) und solchen, bei denen das nicht der Fall war (Strategien 1, 2a-c; $n=21$ ), verglichen ( $t$-Test). Alle statistischen Analysen wurden in der Softwareumgebung R vorgenommen. Um die Relevanz der identifizierten Hindernisse für die Modellierungsprozesse einschätzen zu können, wurde zudem eine kontrastierende Fallanalyse zweier ProbandInnen vorgenommen, bei denen ähnliche Hindernisse identifiziert wurden, die aber verschiedene Modellierungsstrategien umsetzen (Schreier 2010).

\section{Ergebnisse}

\section{Welche Hindernisse lassen sich in den Modellierungsprozessen identifizieren und beschreiben? (Fragestellung 1)}

Innerhalb der Transkripte der ProbandInnen konnten 13 verschiedene Hinderniskategorien identifiziert werden (Tab. 3). 11 der 13 Hinderniskategorien (1-11) werden dabei von den ProbandInnen selbst verbalisiert. Bei den Hinderniskategorien 1 (Fehlende relevante Analogie oder Erfahrung) und 2 (Fehlerhafte Dokumentation) konnten zusätzlich Situationen identifiziert werden, in denen das Hindernis von außen zugeschrieben werden kann. Die Hinderniskategorien 12 (Nichterkennen/Ignorieren von Mängeln am Modell) und 13 (Nichterkennen/Ignorieren von fehlender Passung) werden nur von außen zugeschrieben und beruhen daher nicht auf den Verbalisierungen der ProbandInnen.

Insgesamt wurden 499 Hindernisse kodiert. Im Durchschnitt konnten damit je ProbandIn etwa 14 Hindernisse kodiert werden. Vier der identifizierten Hinderniskategorien kommen bei einzelnen ProbandInnen wiederholt vor. Je ProbandIn konnten drei bis zehn und durchschnittlich etwa sechs verschiedene Hinderniskategorien beobachtet werden. $\mathrm{Zu}$ den wiederholt auftretenden Hinderniskategorien gehören:

- Fehlende relevante Analogie oder Erfahrung ( $n=32$ ProbandInnen mit diesem Hindernis, insgesamt 132 Kodierungen, mittlere Häufigkeit pro ProbandIn $M=3,7$ ),

- Fehlerhafte Dokumentation ( $n=30$, insgesamt $89, M=$ 2,5),

- Hinweise auf unsystematischen Input $(n=27$, insgesamt $61, M=1,7)$ und

- Unzufriedenheit mit den Daten $(n=21$, insgesamt 59, $M=1,6)$.

Andere Hinderniskategorien wie Limitationen bei der Exploration oder Erwartungen an die Aufgabe sind zwar ebenfalls häufig, treten bei den ProbandInnen aber kaum wiederholt auf. Die Hinderniskategorien Randbeobachtung wird überbewertet, Mangelnde Zeichenfähigkeiten, Nichterkennen/Ignorieren von Mängeln am Modell, Nichterkennen/ Ignorieren von fehlender Passung, Zustand des Systems unklar und Material unzureichend wurden insgesamt seltener identifiziert. 
Tab. 3 Kategoriensystem der identifizierten Hindernisse in den Modellierungsprozessen von Biologie-Lehramtsstudierenden $(N=36)$ an einer Blackbox, absteigend sortiert nach Anzahl der ProbandInnen mit den Hindernissen

\begin{tabular}{|c|c|c|c|c|c|}
\hline$\overline{\mathrm{Nr}}$ & Hindernis & Beschreibung & Beispiel & $\begin{array}{l}\text { Anzahl } \\
\text { Proband- } \\
\text { Innen mit } \\
\text { diesem } \\
\text { Hindernis }\end{array}$ & $\begin{array}{l}\text { Anzahl } \\
\text { Kodie- } \\
\text { rungen }\end{array}$ \\
\hline 1 & $\begin{array}{l}\text { Fehlende rele- } \\
\text { vante Analogie } \\
\text { oder Erfahrung }\end{array}$ & $\begin{array}{l}\text { ProbandInnen äußern, dass sie keinerlei Ideen } \\
\text { haben oder nennen gewünschte Wirkung, für } \\
\text { die sie jedoch keine passende Analogie oder } \\
\text { Erfahrung nennen können. } \\
\text { ProbandInnen fällt es schwer sich von bereits } \\
\text { als unzureichend erklärter Idee zu lösen }\end{array}$ & $\begin{array}{l}\text { „Ich habe schon eine Idee, ich } \\
\text { komm nur nicht auf die, wie ich } \\
\text { das, wie genau ich das mache, } \\
\text { damit das so funktioniert, wie ich } \\
\text { mir das vorstelle.“ (Achilles, 50) }\end{array}$ & 32 & 132 \\
\hline 2 & $\begin{array}{l}\text { Fehlerhafte } \\
\text { Dokumentation }\end{array}$ & $\begin{array}{l}\text { ProbandInnen äußern, dass Sie bei der Doku- } \\
\text { mentation einen Fehler gemacht oder etwas } \\
\text { vergessen haben oder sind unsicher über die } \\
\text { Qualität ihrer Dokumentation. } \\
\text { ProbandInnen nehmen eine Beobachtung vor, } \\
\text { aber dokumentieren diese gar nicht oder nicht } \\
\text { richtig. }\end{array}$ & $\begin{array}{l}\text { „Dann habe ich einen Fehler } \\
\text { in meinen Notizen gemacht.“ } \\
(\text { Cynthia, 18) }\end{array}$ & 30 & 89 \\
\hline 3 & $\begin{array}{l}\text { Hinweise auf } \\
\text { unsystemati- } \\
\text { schen Input }\end{array}$ & $\begin{array}{l}\text { ProbandInnen äußern (auch retrospektiv) Pro- } \\
\text { bleme beim Abmessen/Einfüllen der gewünsch- } \\
\text { ten Wassermenge oder begründen die Wasser- } \\
\text { menge willkürlich, z. B. um bestimmte runde } \\
\text { Zahlen zu erreichen. }\end{array}$ & $\begin{array}{l}\text { „D.h. ich habe jetzt eine un- } \\
\text { genaue Menge an Wasser rein } \\
\text { gekippt“ (Floyd, 48) }\end{array}$ & 27 & 61 \\
\hline 4 & $\begin{array}{l}\text { Unzufriedenheit } \\
\text { mit den Daten }\end{array}$ & $\begin{array}{l}\text { ProbandInnen gehen von einem anderen Mus- } \\
\text { ter aus, welches z. B. durch Messfehler und } \\
\text { verschiedene Daten entstanden ist und verfol- } \\
\text { gen dieses weiter, ProbandInnen erkennen kein } \\
\text { Muster, da ihre Datenreihen keine Wiederholun- } \\
\text { gen der Outputs aufweisen. }\end{array}$ & $\begin{array}{l}\text { „Es ist so komisch, dass sich die } \\
\text { Datensätze verändern. Das ist } \\
\text { doch irgendwie/Ich kann einfach } \\
\text { kein Muster erkennen.“ (Cynthia, } \\
68 \text { ) }\end{array}$ & 21 & 59 \\
\hline 5 & $\begin{array}{l}\text { Erwartungen an } \\
\text { die Aufgabe }\end{array}$ & $\begin{array}{l}\text { ProbandInnen äußern die Vermutung die Lö- } \\
\text { sung der Aufgabe sei leichter oder komplexer } \\
\text { als sie zu dem Zeitpunkt annehmen und/oder } \\
\text { nennen andere Erwartungen, die sie nicht zu- } \\
\text { friedenstellend erfüllen können (z. B. mathema- } \\
\text { tische Fähigkeiten). }\end{array}$ & $\begin{array}{l}\text { „Ich hoffe das ist eigentlich kein } \\
\text { Schülerversuch für die } 5 \text { Klasse } \\
\text { oder so und ich beiße mir daran } \\
\text { gerade die Zähne aus.“ (Angeli- } \\
\text { na, 45) }\end{array}$ & 17 & 32 \\
\hline 6 & $\begin{array}{l}\text { Limitationen } \\
\text { bei der Explo- } \\
\text { ration }\end{array}$ & $\begin{array}{l}\text { ProbandInnen kritisieren Beschränkungen im } \\
\text { erwünschten Umgang mit der Blackbox (Öff- } \\
\text { nen, Bewegen, etc.). }\end{array}$ & $\begin{array}{l}\text { „Darf man unten den Schlauch } \\
\text { zuhalten und oben Wasser rein } \\
\text { gießen? T: Nein, bitte nicht. } \\
\text { P: Schade. [stöhnt]“ (Ben, 103) }\end{array}$ & 15 & 18 \\
\hline 7 & $\begin{array}{l}\text { Konkrete Ana- } \\
\text { logie vorhan- } \\
\text { den, deren } \\
\text { Funktionsweise } \\
\text { unbekannt ist }\end{array}$ & $\begin{array}{l}\text { ProbandInnen nennen konkrete Analogie, kön- } \\
\text { nen diese in ihrer Funktionsweise jedoch nicht } \\
\text { erklären oder zeichnen. }\end{array}$ & $\begin{array}{l}\text { „So ein Überlaufschutz, der es } \\
\text { dann komplett entleert. Wie ging } \\
\text { sowas? Wie war denn das?“” } \\
\text { (Jonathan, 41) }\end{array}$ & 13 & 26 \\
\hline 8 & $\begin{array}{l}\text { Zustand des } \\
\text { Systems unklar }\end{array}$ & $\begin{array}{l}\text { Der Zustand des Systems ist den ProbandInnen } \\
\text { unklar, es werden oft Mutmaßungen über be- } \\
\text { reits vor Beginn der Untersuchung enthaltene } \\
\text { Wassermengen aufgestellt. }\end{array}$ & $\begin{array}{l}\text { „Das heißt, das muss irgendwie } \\
\text { auf jeden Fall schon vorher Was- } \\
\text { ser drin gewesen sein.“ (Achilles, } \\
\text { 28) }\end{array}$ & 12 & 15 \\
\hline 9 & $\begin{array}{l}\text { Mangelnde Zei- } \\
\text { chenfähigkeiten }\end{array}$ & $\begin{array}{l}\text { ProbandInnen bekunden Unzufriedenheit mit } \\
\text { ihrer Zeichnung oder bemängeln ihre Zeichen- } \\
\text { fähigkeit im Vorhinein einer Zeichnung und } \\
\text { führen diese infolgedessen gar nicht erst durch. }\end{array}$ & $\begin{array}{l}\text { „Oh ich kann sowas nicht zeich- } \\
\text { nen.“ (Frida, 13) }\end{array}$ & 9 & 16 \\
\hline 10 & $\begin{array}{l}\text { Randbeobachtung } \\
\text { wird überbe- } \\
\text { wertet }\end{array}$ & $\begin{array}{l}\text { ProbandInnen interpretieren Beobachtungen } \\
\text { wie die Fließgeschwindigkeit oder Geräusche } \\
\text { im Inneren der BB als höchst relevant für das } \\
\text { Lösen der Aufgabe und verwenden viel Zeit auf } \\
\text { deren Untersuchung. }\end{array}$ & $\begin{array}{l}\text { „Vielleicht hat das etwas mit } \\
\text { Intensität zu tun.“ (Jim, 8) }\end{array}$ & 8 & 10 \\
\hline
\end{tabular}




\begin{tabular}{|c|c|c|c|c|c|}
\hline $\mathrm{Nr}$ & Hindernis & Beschreibung & Beispiel & $\begin{array}{l}\text { Anzahl } \\
\text { Proband- } \\
\text { Innen mit } \\
\text { diesem } \\
\text { Hindernis }\end{array}$ & $\begin{array}{l}\text { Anzahl } \\
\text { Kodie- } \\
\text { rungen }\end{array}$ \\
\hline 11 & $\begin{array}{l}\text { Material unzu- } \\
\text { reichend }\end{array}$ & $\begin{array}{l}\text { ProbandInnen wünschen sich zusätzliche Mate- } \\
\text { rialien oder kritisieren vorhandenes Material. }\end{array}$ & $\begin{array}{l}\text { „Um das jetzt zu testen, ohne } \\
\text { das Gerät zu öffnen, würde ich } \\
\text { sowas ähnliches bauen, aber ich } \\
\text { sehe jetzt irgendwie nicht so } \\
\text { Materialien, die sich eignen.“ } \\
\text { (James, 25) }\end{array}$ & 8 & 19 \\
\hline 12 & $\begin{array}{l}\text { Nichterkennen/ } \\
\text { Ignorieren von } \\
\text { Mängeln am } \\
\text { Modell }\end{array}$ & $\begin{array}{l}\text { ProbandInnen erkennen physikalische Mängel } \\
\text { nicht oder ignorieren diese und behalten sie } \\
\text { aufgrund fehlender Alternativideen bei. }\end{array}$ & $\begin{array}{l}\text { „da müssten die Behälter ja ir- } \\
\text { gendwann voll sein Mist!““ (igno- } \\
\text { riert und macht weiter; Jenny, } \\
\text { 49) }\end{array}$ & 4 & 8 \\
\hline 13 & $\begin{array}{l}\text { Nichterkennen/ } \\
\text { Ignorieren } \\
\text { von fehlender } \\
\text { Passung }\end{array}$ & $\begin{array}{l}\text { Physikalische oder datenbasierte Nichtpassung } \\
\text { werden von den ProbandInnen nicht erkannt } \\
\text { oder bewusst aufgrund fehlender Alternative } \\
\text { beibehalten. }\end{array}$ & $\begin{array}{l}\text { „Eigentlich kann ich das verwer- } \\
\text { fen, weil dazu passt mein Modell } \\
\text { nicht.“ (verwirft Modell jedoch } \\
\text { nicht; Cynthia, 33) }\end{array}$ & 4 & 10 \\
\hline
\end{tabular}

\section{Inwiefern treten die identifizierten Hindernisse in einzelnen Phasen der Modellierungsprozesse besonders häufig auf? (Fragestellung 2)}

Abb. 3 zeigt, wie viele Kodierungen je Hinderniskategorie mit den identifizierten Tätigkeiten (Tab. 1) zusammen auftreten. Die meisten Hindernisse treten zusammen mit der Tätigkeit Zusammenfassung auf, wobei insbesondere die Hindernisse Fehlerhafte Dokumentation, Hinweise auf unsystematischen Input, Unzufriedenheit mit den Daten und Fehlende relevante Analogie oder Erfahrung hier häufig identifiziert werden. Das Hindernis Fehlende relevante Analogie oder Erfahrung tritt zudem mit allen Tätigkeiten zusammen auf, die der Exploration und Herstellung zugeordnet werden, und darüber hinaus mit der Tätigkeit Hypothese falsifiziert, die der Phase der Vorhersage zugeordnet wird. Besonders häufig tritt das Hindernis Fehlende relevante Analogie oder Erfahrung dabei zusammen mit den Tätigkeiten Beobachtung eines Phänomens, Prüfung Konsistenz Modell/Daten, Zusammenfassung und Analogien und Erfahrungen auf. Alle identifizierten Hindernisse treten zudem mindestens einmal während der Tätigkeit Prüfung Konsistenz Modell/Daten auf.

Abb. 2 zeigt die relative Häufigkeit (prozentual) der identifizierten Hindernisse in den drei Phasen des Modellierungsprozesses: Exploration, Herstellung und Vorhersage. Innerhalb der Modellierungsprozesse wurden die meisten Hindernisse in den Phasen der Exploration (299) oder der Herstellung (131) identifiziert, die auch einen Großteil des beobachtbaren Verhaltens ausmachen (68\% Tätigkeiten der Exploration, $28 \%$ Tätigkeiten der Herstellung). Selten wurden Hindernisse dagegen in der Phase der Vorhersage (21) identifiziert, die insgesamt allerdings auch selten vorkommen. Dabei zeigen sich hindernisspezifische Unter- schiede über die Phasen der Modellierungsprozesse hinweg. So treten zum Beispiel die Hinderniskategorien Hinweise auf unsystematischen Input und Fehlerhafte Dokumentation primär in Phasen der Exploration auf, wohingegen die Hinderniskategorien konkrete Analogie vorhanden, deren genaue Funktionsweise unbekannt ist, Nichterkennen/ ignorieren von Mängeln am Modell und Nichterkennen/ ignorieren von fehlender Passung vor allem in Phasen der Herstellung auftreten. Die Hinderniskategorie Mangelnde Zeichenfähigkeit tritt ausschließlich in der Phase der Herstellung auf. Keine der Hinderniskategorien tritt anteilig am häufigsten in der Phase der Vorhersage auf. Grundsätzlich ergeben sich die gleichen Befunde, wenn auch die zeitliche Dauer der Phasen berücksichtigt wird (Anhang, Abb. 7).

\section{Inwiefern besteht ein Zusammenhang zwischen den identifizierten Hindernissen und den umgesetzten Modellierungsprozessen? (Fragestellung 3)}

Abb. 4 zeigt die Häufigkeit der Hindernisse in Abhängigkeit der umgesetzten Modellierungsstrategie. Für die häufigste Hinderniskategorie Fehlende relevante Analogie oder Erfahrung liegt der Median der ( $z$-standardisierten) Häufigkeit beispielsweise bei Med=-0,62 (Strategie 2a), Med=0,31 (Strategie 2b), Med=0,12 (Strategie 2c), Med=0,49 (Strategie 3a), Med=0,12 (Strategie 3b). Dies ist die einzige Hinderniskategorie, bei der sich nach Zusammenfassung von ProbandInnen, die keine Tätigkeiten der Vorhersage umsetzen (Med $=-0,62 ; n=21)$ und solchen, die Tätigkeiten der Vorhersage umsetzen (Med $=0,49 ; n=15)$, augenscheinlich ein Häufigkeitsunterschied zwischen den Gruppen ergibt (Abb. 4, unten).

Statistisch zeigen sich keine signifikanten Unterschiede hinsichtlich der Häufigkeit der Hindernisse zwischen den 


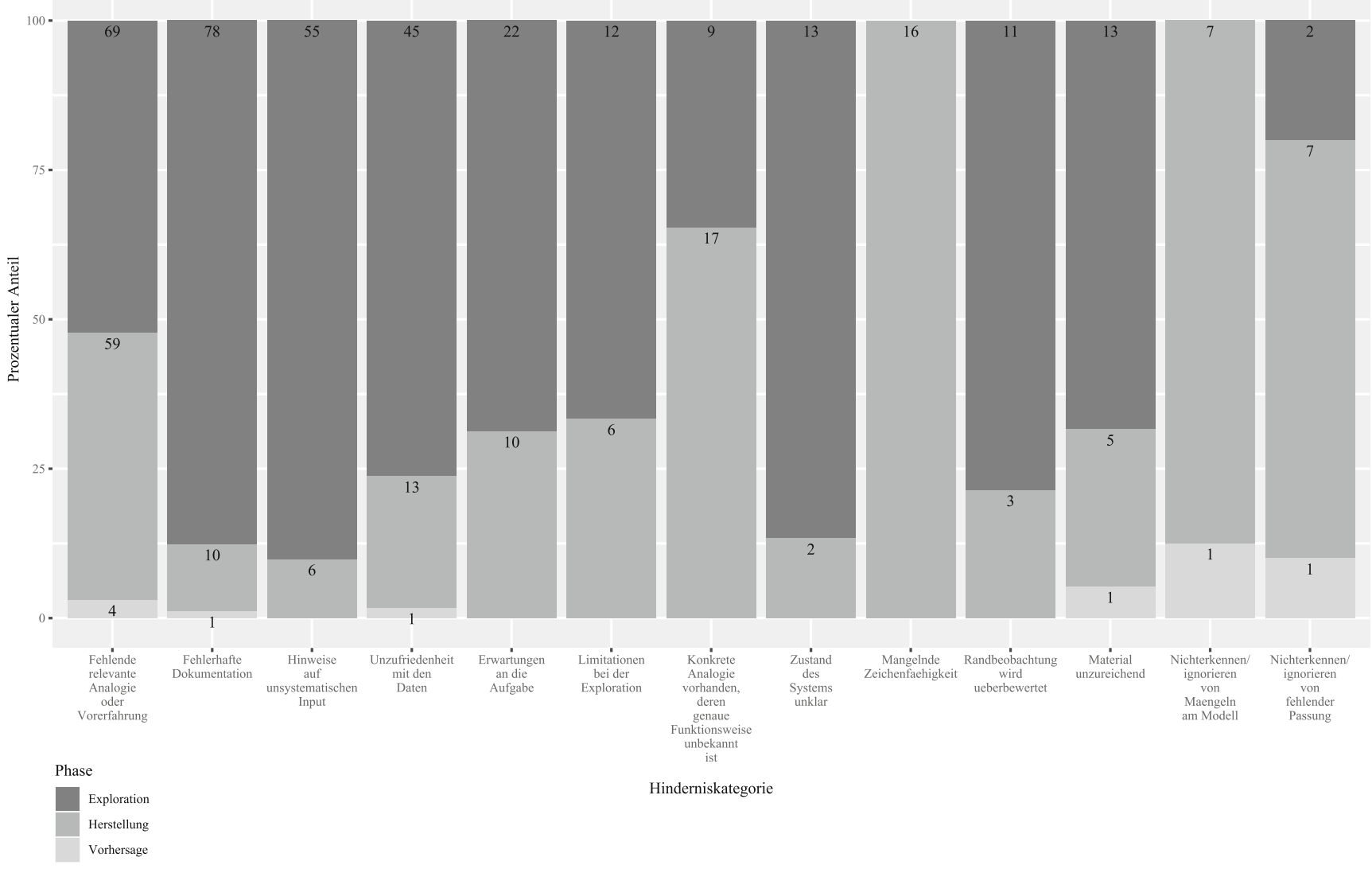

Abb. 2 Gestapeltes Säulen-Diagramm aller Hindernisse unterteilt nach ihrem Auftreten in den Phasen des Modellierungsprozesses. Zur besseren Sichtbarkeit der Verhältnisse wurden die relativen (prozentualen) Häufigkeiten dargestellt

sechs Modellierungsstrategien (ANOVAs; $p>0,05$ für alle Hindernisse). Auch der Vergleich von ProbandInnen, die Tätigkeiten der Vorhersage umsetzen und solchen, die keine Tätigkeiten der Vorhersage umsetzen, zeigt keine signifikanten Unterschiede in der Häufigkeit der Hindernisse ( $t$ Test; $p>0,05)$.

Über die statistische Analyse hinaus liefert die Fallanalyse Evidenz für die Relevanz der identifizierten Hindernisse für die Modellierungsprozesse der ProbandInnen (Schreier 2010). Im Folgenden werden daher die ProbandInnen James und Jasmin im Detail betrachtet. Diese zeigen ähnliche Hindernisse, allerdings sehr unterschiedliche Modellierungsprozesse (Tab. 4).

Die Modellierungsprozesse von James und Jasmin sind in Abb. 5 als Codelines illustriert. Diese Codelines zeigen die event-basiert kodierten Tätigkeiten des Modellierens. Hierbei wird die zeitliche Abfolge der Tätigkeiten als Events auf der x-Achse und die jeweils kodierte Tätigkeit auf der y-Achse dargestellt. Die kodierten Hindernisse und ihr Auftreten eingebettet in die Tätigkeiten sind als Sprechblasen dargestellt, sodass ersichtlich wird, wo in den beiden Modellierprozessen, welche Hindernisse aufgetreten sind. Der Modellierungsprozess von James besteht aus 52 kodierten Tätigkeiten und dauert etwa 27 min (Abb. 5,
Tab. 4 Übersicht über die Häufigkeit der Hindernisse bei James und Jasmin

\begin{tabular}{llll}
\hline $\mathrm{Nr}$ & Problemkategorie & James & Jasmin \\
\hline 1 & $\begin{array}{l}\text { Fehlende relevante Analogie oder Er- } \\
\text { fahrung }\end{array}$ & 3 & 1 \\
2 & Fehlerhafte Dokumentation & 1 & 1 \\
3 & Hinweise auf unsystematischen Input & 2 & 3 \\
4 & Unzufriedenheit mit den Daten & - & 2 \\
5 & Erwartungen an die Aufgabe & - & 1 \\
6 & Limitationen bei der Exploration & - & - \\
7 & Konkrete Analogie vorhanden, deren & - & - \\
& Funktionsweise unbekannt ist & & \\
9 & Mangelnde Zeichenfähigkeiten & - & - \\
10 & Randbeobachtung wird überbewertet & - & - \\
10 & Zustand des Systems unklar & 1 & - \\
11 & Material unzureichend & 3 & 2 \\
12 & Nichterkennen/Ignorieren von Mängeln & - & - \\
& am Modell & & \\
13 & Nichterkennen/Ignorieren von fehlen- & - & - \\
& der Passung & & \\
- & Total & 10 & 10 \\
\hline
\end{tabular}




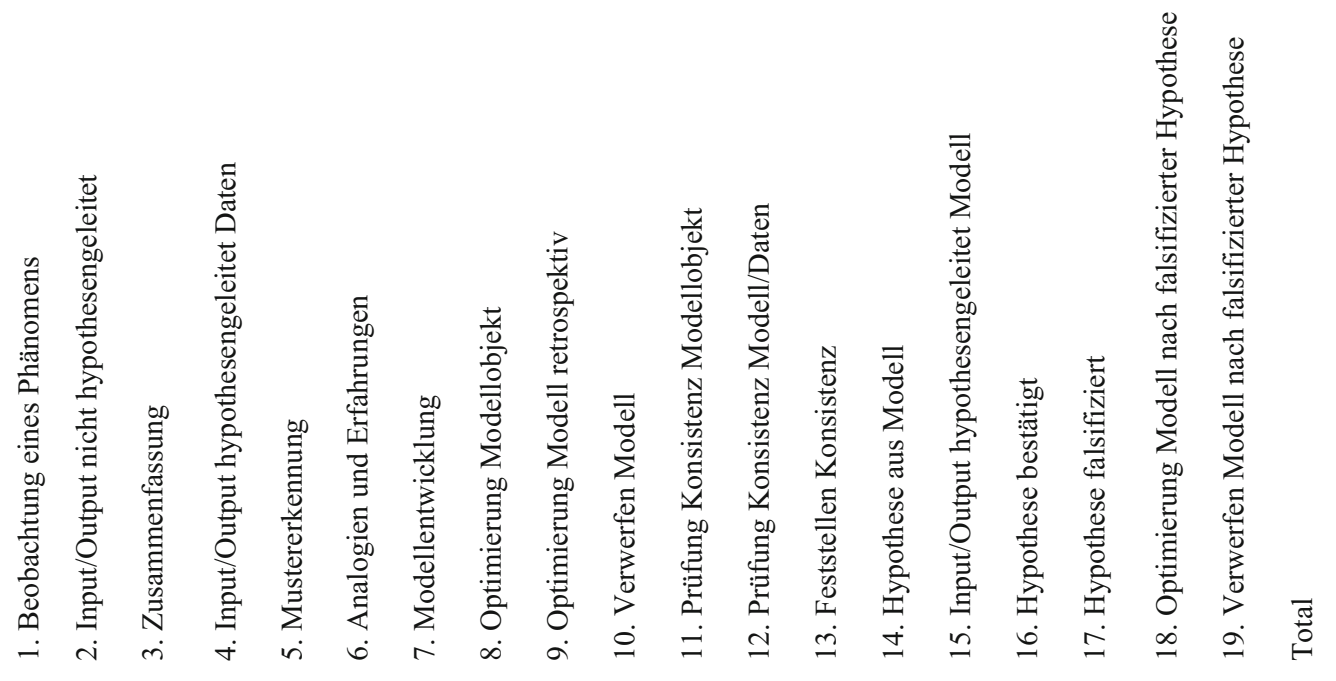

1 Fehlende relevante

Analogie oder Erfahrung

$\begin{array}{lll}30 & 4 & 19\end{array}$

$\begin{array}{llll}3 & 16 & 8\end{array}$

24

$12 \quad 25 \quad 2$

\begin{tabular}{|c|c|c|c|c|c|c|c|c|c|c|c|c|c|c|c|c|c|c|c|c|}
\hline $\begin{array}{l}2 \text { Fehlerhafte } \\
\text { Dokumentation }\end{array}$ & 10 & 14 & 42 & 11 & 0 & 2 & 0 & 0 & 1 & 1 & 2 & 4 & 0 & 1 & 1 & 0 & 0 & 0 & 0 & 89 \\
\hline $\begin{array}{l}3 \text { Hinweise auf } \\
\text { unsystematischen Input }\end{array}$ & 6 & 12 & 21 & 13 & 0 & 1 & 0 & 0 & 0 & 2 & 1 & 3 & 0 & 0 & 2 & 0 & 0 & 0 & 0 & 61 \\
\hline $\begin{array}{l}4 \text { Unzufriedenheit mit den } \\
\text { Daten }\end{array}$ & 18 & 3 & 19 & 4 & 0 & 1 & 1 & 0 & 1 & 2 & 0 & 9 & 0 & 0 & 0 & 0 & 1 & 0 & 0 & 59 \\
\hline $\begin{array}{l}5 \text { Erwartungen an die } \\
\text { Aufgabe }\end{array}$ & 7 & 3 & 8 & 3 & 0 & 4 & 0 & 0 & 1 & 3 & 0 & 2 & 1 & 0 & 0 & 0 & 0 & 0 & 0 & 32 \\
\hline $\begin{array}{l}6 \text { Limitationen bei der } \\
\text { Exploration }\end{array}$ & 1 & 1 & 7 & 1 & 0 & 1 & 1 & 0 & 0 & 2 & 0 & 3 & 0 & 0 & 1 & 0 & 0 & 0 & 0 & 18 \\
\hline
\end{tabular}

7 Konkrete Analogie

vorhanden, deren

Funktionsweise unbekannt

ist

8 Zustand des Systems unklar

9 Mangelnde

Zeichenfähigkeiten

10 Randbeobachtung wird

überbewertet

\begin{tabular}{lllllllllllllllllllllll}
\hline 1 Material unzureichend & 1 & 4 & 7 & 1 & 0 & 1 & 0 & 0 & 0 & 0 & 1 & 3 & 0 & 0 & 0 & 1 & 0 & 0 & 0 & 19 \\
\hline
\end{tabular}

12 Nichterkennen/Ignorieren

von Mängeln am Modell

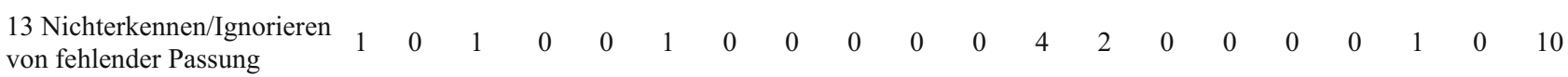
Ton fehlender Passung

Total

$\begin{array}{llllllllllllllllllll}76 & 47 & 137 & 38 & 1 & 48 & 23 & 3 & 9 & 13 & 21 & 66 & 5 & 1 & 4 & 1 & 4 & 2 & 0 & 499\end{array}$

Abb. 3 Kreuztabelle der Hinderniskategorien und Tätigkeiten aller 36 ProbandInnen, die zeigt, wie viele Kodierungen je Hinderniskategorie mit den identifizierten Tätigkeiten gemeinsam auftreten 


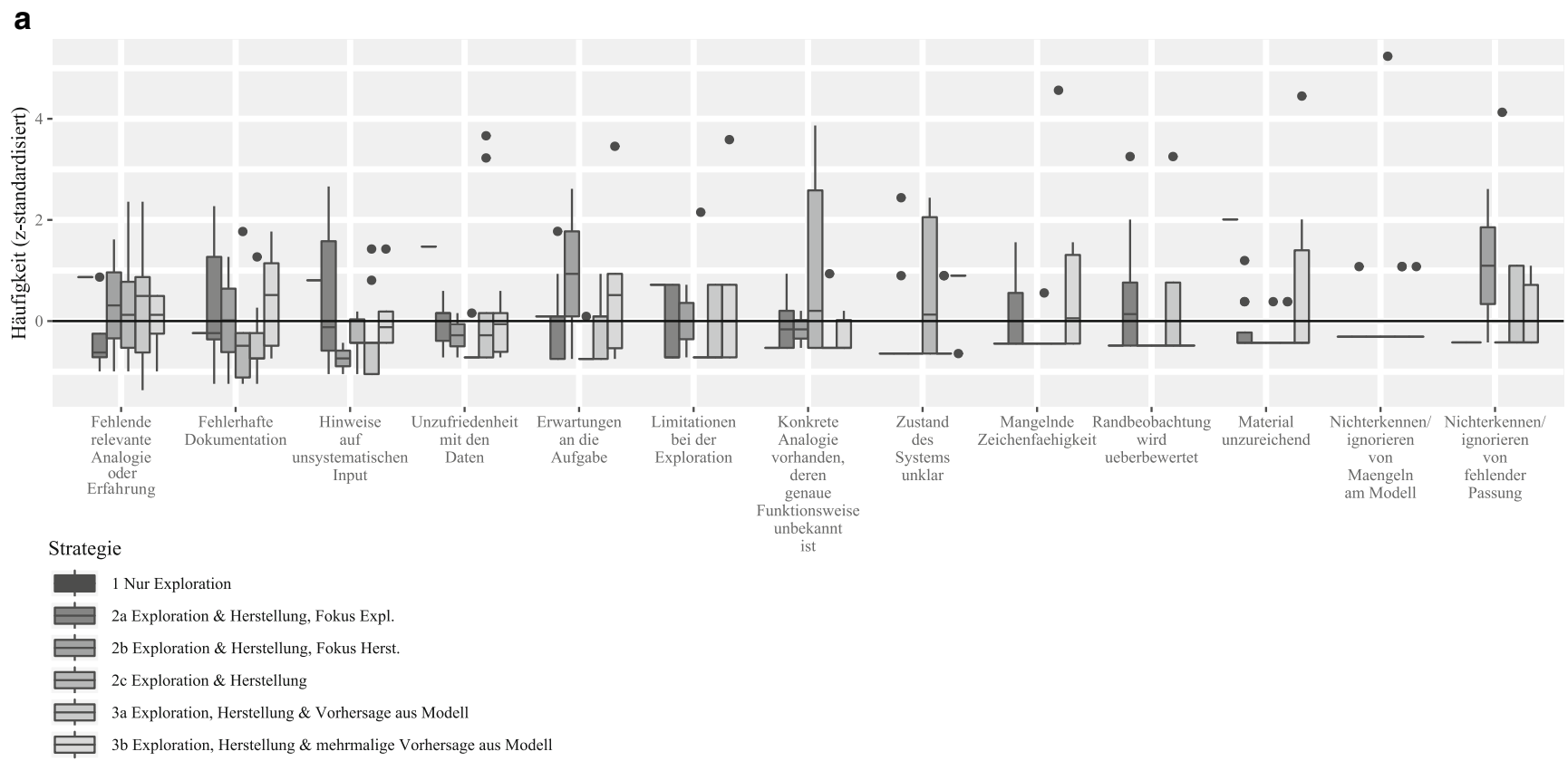

b

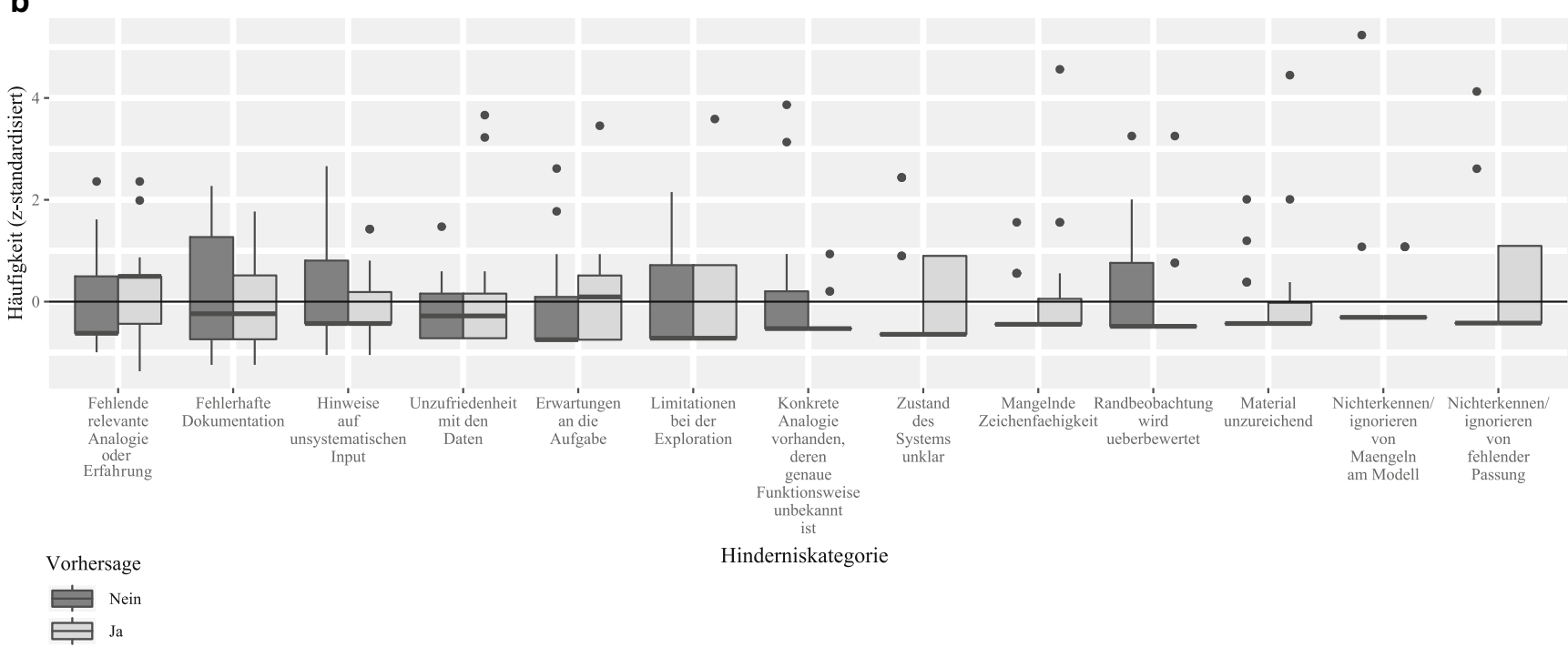

Abb. 4 Boxplots der $z$-standardisierten Häufigkeit der Problemkategorien. a gruppiert nach Modellierungsstrategie (vgl. Tab. 2); b gruppiert basierend auf dem (Nicht-)Auftreten der Tätigkeit 14: „Probanden nutzen Modell, um Vorhersage über Output bei bestimmtem Input zu treffen“

oben). Hierbei durchläuft James in seinem Modellierungsprozess alternierend Phasen der Exploration, Herstellung und Vorhersage (Modellierungsstrategie 3b; vgl. Tab. 2). In James' Modellierungsprozess treten zehn Hindernisse aus sechs Hinderniskategorien auf, die sich relativ gleichmäßig auf die drei Phasen der Modellierung verteilen. Der Modellierungsprozess von Jasmin dauert $1 \mathrm{~h}$ und $28 \mathrm{~min}$ und besteht aus 71 kodierten Tätigkeiten (Abb. 5, unten). Jasmins Modellierungsprozess setzt sich hauptsächlich aus Tätigkeiten der Exploration zusammen, sowie fünf kürzeren Phasen der Herstellung, in denen sie ihr Modell entwickelt (Modellierungsstrategie 2a; vgl. Tab. 2). In Jasmins Model- lierungsprozess wurden zehn Hindernisse aus sechs Hinderniskategorien identifiziert.

James Die ersten Hindernisse treten bei James auf als er, ohne einen spezifischen Grund zu nennen, die Menge des Inputs von vorher $400 \mathrm{ml}$ auf $200 \mathrm{ml}$ verringert (Anhang, Tab. 6; Hinderniskategorie 3) und einen Output von $350 \mathrm{ml}$ beobachtet (Events 13, 14). Diese Beobachtung eines Outputs, der größer ist als die Input-Wassermenge, veranlasst ihn dazu, sich Gedanken über den Ausgangszustand der Blackbox zu machen (,Also insgesamt kann natürlich im gesamten System schon vorher mehr Flüssigkeit drin ge- 


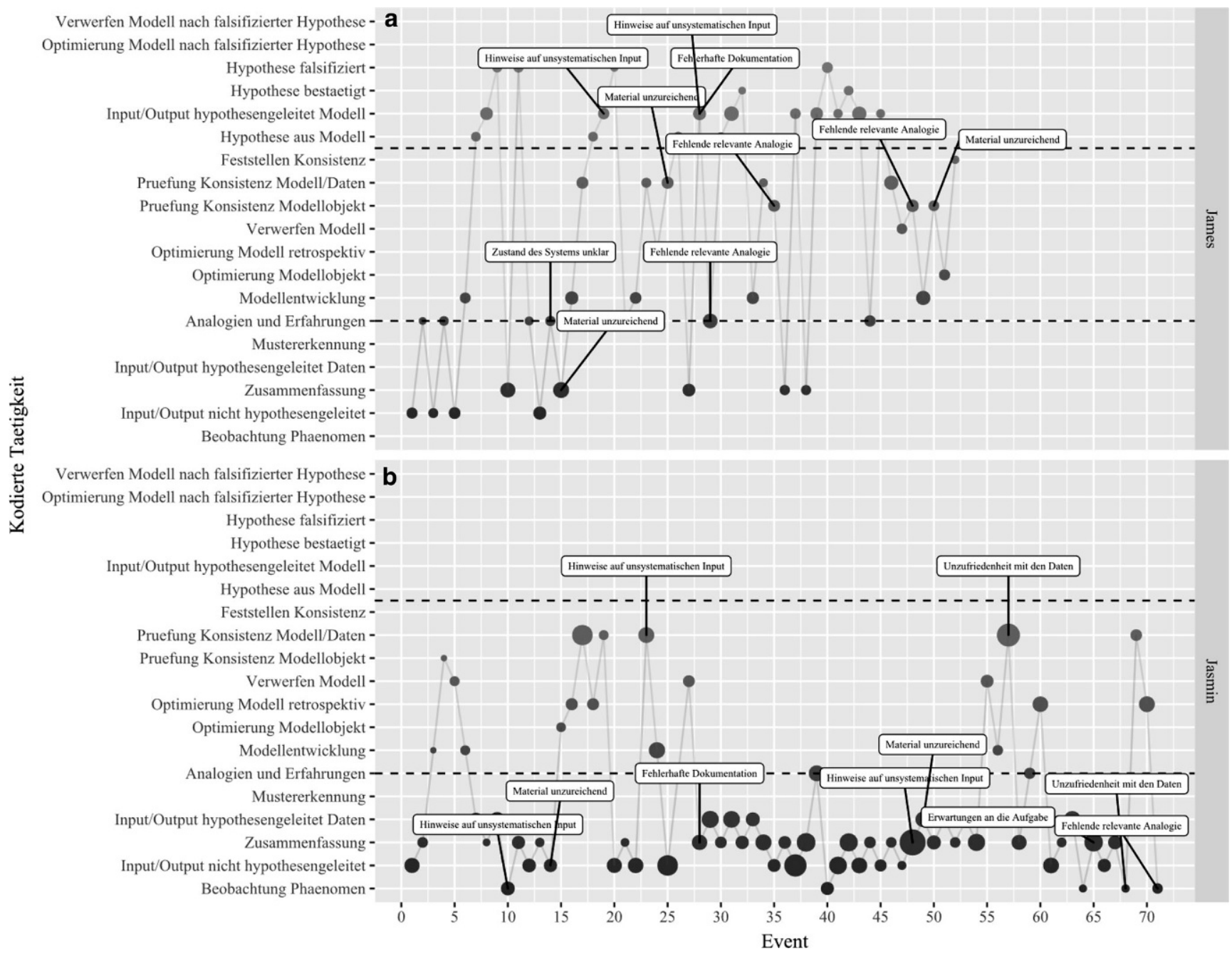

Abb. 5 Tätigkeitssequenzen der ProbandInnen James (a) und Jasmin (b), die das jeweilige Vorgehen im Modellierungsprozess sowie die darin identifizierten Hindernisse zeigen. Die Hinderniskategorie „Fehlende relevante Analogie oder Vorerfahrung“ ist aus Gründen der besseren Lesbarkeit als „Fehlende relevante Analogie“ abgekürzt

wesen sein.“; Hinderniskategorie 8). Zudem äußert er den Wunsch, nicht nur ein ,theoretisches“, sondern ein gegenständliches Modell entwickeln zu können, für welches allerdings keine Materialien vorliegen (Hinderniskategorie 11). Stattdessen hantiert er mit den verfügbaren Wassergläsern, um sich vor Augen zu führen, ,was passiert, wenn zwei verschiedene Gefäße mit unterschiedlicher Menge Wasser gefüllt werden“, wobei er sich in seiner Annahme bestätigt, dass ,auch immer nur so viel rauskommen [kann], wie eigentlich auch reinkommt." James zeichnet nun ein weiteres Modell (Event 16), in welchem er die bisherige Zeichnung zweier Gefäße um eine Wippe erweitert, die er retrospektiv anhand von auditiven Beobachtungen evaluiert (,Das hörte sich eher an, wie so ein Schlauch, der befüllt wird."). Um diese Hypothese zu prüfen, gießt er anschließend eine gröBere Menge Wasser ein, die er nicht genauer abmisst (,Jetzt ist die Menge des Wassers erstmal egal, das ich hineinfülle."; Hinderniskategorie 3) und verwirft die Analogie einer
Wippe auf Basis seiner Beobachtung. James zieht nun ausgehend von der Beobachtung, dass bei jedem Output das Wasser eher schwallartig herausfließt, weitere Analogien heran, aus denen er ein weiteres Modell entwickelt (Events 22-25). Hierbei integriert er zuerst eine Membran, die langsames Diffundieren des Wassers in ein weiteres Gefäß mit einem Kippmechanismus erlaubt. Zur Überprüfung dieses Modells möchte er erneut ein gegenständliches Modell entwickeln, stellt aber fest: „Ich sehe jetzt irgendwie nicht so Materialien, die sich eignen." (Hinderniskategorie 11). Bei der weiteren Exploration der Blackbox variiert James nun weiterhin die Inputmengen (Hinderniskategorie 3), um beispielsweise zu sehen, ,was passiert, wenn ich nur eine sehr geringe Menge Wasser hineingebe" und reflektiert, dass er nicht dokumentiert oder abmisst, wie viel Wasser er hineingibt (Hinderniskategorie 2). Außerdem erwartet er, dass es zum Inneren der Blackbox eine ,Entsprechung in der Natur [gibt], die [ihm] jetzt gerade noch nicht einfällt" (Hinder- 
niskategorie 1). In der Evaluation seines finalen Modells, welches erneut einen Wippenmechanismus beinhaltet (Anhang, Abb. 8), sagt er: „Leider fällt mir kein anderer Mechanismus ein [...], da bin ich jetzt auch kein Ingenieur, Techniker oder Hydrauliker, da fehlt mir einfach ein bisschen Hintergrundwissen." (Hinderniskategorie 1).

Jasmin In Jasmins Modellierungsprozess wurden 10 Hindernisse identifiziert, die vornehmlich in Situationen auftreten, in denen sie ihre Beobachtungen zusammenfasst (Tätigkeit 3, vgl. Tab. 1) oder ihr Modell retrospektiv mit beobachteten Daten vergleicht (Tätigkeit 13). Die ersten Hindernisse treten bei Jasmin in der zweiten Explorationsphase (Events 7-14) auf, als sie einen Output beobachtet, den sie, basierend auf ihren vorherigen Beobachtungen, nicht erwartet hat. Hierbei nimmt sie den beobachteten Output nicht als valides Ergebnis ihrer Untersuchung wahr, sondern schließt darauf, dass sie selbst unsystematisch vorgegangen ist (,Also habe ich das falsch abgemessen."; Hinderniskategorie 3) oder die Blackbox nicht funktionsfähig ist (,Ich glaube das Gerät ist kaputt"; Hinderniskategorie 11). Tatsächlich jedoch beginnt sie ab dieser Situation, in ihrem Inputverhalten unsystematisch zu werden und variiert die nächsten Inputmengen mit dem Ziel, bestimmte, von ihr erwartete Outputs zu beobachten (Anhang, Tab. 6; Inputs 4-10). In Jasmins Modellierungsprozess können ab hier weitere Hindernisse beobachtet werden: Teilweise werden Beobachtungen nicht dokumentiert (Hinderniskategorie 2), sie äußert wiederholt, beim Abmessen ungenau vorgegangen zu sein (Hinderniskategorie 3) und begründet dies mit unzureichendem Material (,Ich hätte jetzt liebend gern eine Pipette.“; Hinderniskategorie 11). Am Ende ihres Modellierungsprozesses ist sie entsprechend unzufrieden mit den von ihr aufgenommenen Daten (Hinderniskategorie 4), obwohl sie von sich selbst erwartet, die Aufgabe lösen zu können: „Ich denke für so einen Kram viel zu kompliziert. Meistens ist das so eine banale Billiglösung am Ende." Die Grundidee aufeinanderfolgender Gefäße behält sie über ihren gesamten Modellierungsprozess hin bei, wobei sie die Darstellungsweise sowie Anzahl und Volumina der Gefäße ändert, bis sie bei ihrem finalen Modell angekommen ist (Anhang, Abb. 8). Letztlich beschließt sie, dass ihr eine passende Idee fehlt (Hinderniskategorie 1), und beendet frustriert ihren Modellierungsprozess (,Ich hab[e] keinen Plan [und] auch keinen Bock mehr. [...] [Ich] komm[e] nicht zu einer Lösung [...]. Den Rest überlasse ich dann den Profis.“).

Zusammenfassend zeigt eine qualitative Betrachtung der beiden ProbandInnen James und Jasmin, dass diese zwar durchaus ähnliche Hindernisse in ihren Modellierungsprozessen erfahren und verbalisieren, beide jedoch unterschiedlich mit diesen Hindernissen umgehen. Während James beim Auftreten von Hindernissen sein Modell verändert und wiederholt testet, konzentriert sich Jasmin auf die weitere Exploration der Blackbox, in der Hoffnung, die erwarteten Daten zu generieren.

\section{Diskussion}

Die vorliegende Studie hatte zum Ziel, Hindernisse zu identifizieren und zu beschreiben, die in Modellierungsprozessen von Lehramtsstudierenden bei der Untersuchung einer Blackbox auftreten. Im Folgenden werden die Ergebnisse in Bezug auf die drei Fragestellungen diskutiert.

\section{Welche Hindernisse lassen sich in den Modellierungsprozessen identifizieren und beschreiben? (Fragestellung 1)}

Es wurden 13 Hinderniskategorien in den Modellierungsprozessen der 36 ProbandInnen identifiziert (Tab. 3). Hierbei konnten teilweise Hinderniskategorien repliziert werden, die bereits in anderen Forschungsarbeiten zum Modellieren (Hogan und Thomas 2001; Meister et al. 2020; Sins et al. 2005; Windschitl und Thompson 2006), Experimentieren (Baur 2018; Hammann 2004), sowie wissenschaftlichen Arbeiten allgemein (Woolley et al. 2018) identifiziert worden waren (Hinderniskategorien 2 (Fehlerhafte Dokumentation), 3 (Hinweise auf unsystematischen Input), 4 (Unzufriedenheit mit den Daten) und 10 (Randbeobachtung wird überbewertet)). Parallelen zum Experimentieren und wissenschaftlichen Arbeiten im Allgemeinen lassen sich insbesondere im Hinblick auf die explorativen Tätigkeiten des Modellierens und den Umgang mit den generierten Daten erkennen: Unsystematisches Vorgehen (Baur 2018; Hammann 2004; Woolley et al. 2018), die Auswahl unvollständiger oder unrelevanter Variablen (Hammann 2004; Woolley et al. 2018) und die fehlerhafte Dokumentation von Ergebnissen (Woolley et al. 2018) können arbeitsweisenunabhängig und über verschiedene Zielgruppen hinweg beobachtet werden.

Die Hinderniskategorien 1 (Fehlende relevante Analogie oder Erfahrung), 7 (Konkrete Analogie vorhanden, deren Funktionsweise unbekannt ist), 12 (Nichterkennen/ Ignorieren von Mängeln am Modell) und 13 (Nichterkennen/Ignorieren von fehlender Passung) scheinen dagegen spezifisch für das Modellieren zu sein, da sie nicht in der zum Vergleich herangezogenen Studien zum Experimentieren (Baur 2018; Hammann 2004) oder wissenschaftlichen Arbeiten allgemein (Woolley et al. 2018) beschrieben werden. Die zentrale Rolle von Analogien für Modellierungen (Clement 2008; Krell et al. 2019) wird insbesondere durch das häufige Auftreten der Hinderniskategorie 1 deutlich. Gleichzeitig zeigt Hinderniskategorie 7, dass einige ProbandInnen, analog $\mathrm{zu}$ den Beobachtungen von Windschitl und Thompson (2006), Probleme haben, 
richtig erkannte Zusammenhänge in ihre Modelle zu integrieren. Beide Hinderniskategorien stützen hierbei die Vermutung, dass Modellieren und deklaratives Fachwissen in einem korreliert-abhängigen Verhältnis stehen (vgl. Nehring und Schwichow 2020). Dies wird exemplarisch in James' Verbalisierungen bezüglich seines finalen Modells deutlich (,Leider fällt mir kein anderer Mechanismus ein [...], da bin ich jetzt auch kein Ingenieur, Techniker oder Hydrauliker, da fehlt mir einfach ein bisschen Hintergrundwissen."). Das Verhältnis zwischen Hinderniskategorie 1 und 7 könnte im weitgehenden Fehlen von relevantem, deklarativem Fachwissen zur Blackbox begründet sein. Mangelndes Fachwissen, fehlende relevante Analogien oder Erfahrungen oder geringe Kreativität kann das für Modellierungen zentrale abduktive Schließen behindern, also die erkenntniserweiternde Entwicklung erklärender Modelle (Magnani 1999; Krüger und Upmeier zu Belzen 2021). Die Hinderniskategorien 4 (Unzufriedenheit mit den Daten), 12 (Nichterkennen/Ignorieren von Mängeln am Modell) und 13 (Nichterkennen/Ignorieren von fehlender Passung) deuten zudem darauf hin, dass der Wechsel zwischen Modell- und Erfahrungswelt, das heißt die Vermittlung zwischen Modell und generierten Daten (DounasFrazer et al. 2018), problematisch wird, wenn die ProbandInnen die beobachteten Daten nicht interpretieren können oder als unlogisch erachten (Hammann 2004). Solche unerwarteten, anomalen Daten, welche die Blackbox durch ihren inneren Mechanismus provoziert (insb. wenn der Output das eingefüllte Volumen eines Inputs überschreitet), werden oftmals nicht anhand eines gezeichneten Modells erklärt, sondern vielmehr methodisch unter anderem mit mangelnden eigenen Fähigkeiten begründet (Meister et al. 2020).

Obwohl Hinderniskategorie 9 (Mangelnde Zeichenfähigkeit) in keiner der zum Vergleich herangezogenen Studien zum Modellieren (Hogan und Thomas 2001; Meister et al. 2020; Sins et al. 2005; Windschitl und Thompson 2006), Experimentieren (Baur 2018; Hammann 2004) oder wissenschaftlichen Arbeiten allgemein (Woolley et al. 2018) beschrieben wird, weisen andere Studien durchaus auf die Relevanz des Zeichnens sowie damit verbundenen Hindernissen im Kontext naturwissenschaftlichen Unterrichts hin (Chittleborough und Treagust 2007; Quillin und Thomas 2015). Bei einem Probanden (Carlo) ist Mangelnde Zeichenfähigkeit als Hindernis besonders auffällig: Er zeichnet überhaupt kein Modell und begründet dies sowohl mit mangelnden Fähigkeiten als auch geringer Motivation, wodurch sein Modellierungsprozess nur aus Tätigkeiten der Exploration besteht (Strategie 1; Tab. 2). Studien aus der Repräsentationsforschung bieten hier zwei mögliche Erklärungen: Zum einen ist der Proband eventuell kein ,Visualisierer“ (Koć-Januchta et al. 2017), sodass ihm das Zeichnen als nicht hilfreich zur Lösung der Aufgabe erscheint. Zum anderen könnte er das Zeichnen aktiv vermeiden um kein „fehlerhaftes“ Modell als Endergebnis präsentieren zu müssen. Eine weitere Probandin (Iris) verwirft beispielsweise ihr finales Modell, da sie es als falsch einschätzt und nicht als Ergebnis präsentieren möchte. Bei beiden ProbandInnen wird damit zusätzlich sichtbar, dass volitionale Aspekte (z. B. Selbstregulation, Selbstwirksamkeitserwartung; Bandura 1994) bei Modellierungsprozessen eine große Rolle spielen (Hogan und Thomas 2001; Nehring et al. 2015; Nordheimer 2019). Motivationale und volitionale Aspekte sind zwar Teil gängiger Kompetenzdefinitionen (Weinert 2001), werden jedoch in der Kompetenzforschung oft explizit ausgeklammert (Klieme et al. 2007). Insbesondere zu Kompetenzen naturwissenschaftlicher Erkenntnisgewinnung und des Modellierens liegen keine prozessorientierten Studien vor, die motivationale und volitionale Aspekte als Einflussfaktoren auf die Performanz (d.h. das Modellieren) untersuchen. Zukünftige Studien könnten hier anknüpfen und beispielsweise die Selbstregulation oder Selbstwirksamkeitserwartung während des Modellierens untersuchen (Bandura 1994; Nordheimer 2019).

Die weiteren Hinderniskategorien und 5 (Erwartungen an die Aufgabe), 6 (Limitationen bei der Exploration), 8 (Zustand des Systems unklar) und 11 (Material unzureichend) scheinen dagegen im konkreten Setting begründet zu sein. Das Auftreten der Hinderniskategorien 6 (Limitationen bei der Exploration) und 8 (Zustand des Systems unklar) könnte gerade im Zusammenhang mit den Hinderniskategorien 1 (Fehlende relevante Analogie oder Erfahrung) und 4 (Unzufriedenheit mit den Daten) dazu führen, dass die Erfahrungswelt nicht als ausreichend fruchtbar wahrgenommen wird, um einen Transfer in die Modellwelt zu ermöglichen, wenn zum Beispiel das Erkennen einer Regelmäßigkeit in den Daten ausbleibt (Leisner-Bodenthin 2006). Im Hinblick auf Hinderniskategorien 6 (Limitationen bei der Exploration) und 8 (Zustand des Systems unklar) und im Kontext von Modellieren als Problemlösen kann die Blackbox als komplexes, intransparentes Problem verstanden werden (Funke 2011). Es ist somit erwartbar, dass ähnliche Hindernisse auch bei anderen, vergleichbar intransparenten Problemen beobachtet werden können. Insgesamt stützen solche spezifisch im Setting begründeten Hindernisse theoretische Annahmen zur Abhängigkeit der Performanz von der Aufgabenstellung und von situationsspezifischen Fähigkeiten (Blömeke et al. 2015). Dies wird auch in den Hinderniskategorien 11 (Material unzureichend) und 13 (Erwartungen an die Aufgabe) deutlich. Insbesondere Hinderniskategorie 13 zeigt, dass die Wahrnehmung der Situation (vgl. Blömeke et al. 2015) den Modellierungsprozess beeinflussen kann: Einige ProbandInnen (Boris, Kara; Anhang, Tab. 5) verbalisieren bereits nach wenigen Minuten, die Blackbox ,gelöst“ zu haben und möchten daraufhin aufhören. Das Setting wird als kur- 
ze, vollständig lösbare Aufgabe und nicht als komplexes Problem betrachtet (Hogan und Thomas 2001; Schauble et al. 1991). Berland et al. (2016) argumentieren hierzu, dass wissenschaftliche Praxis (hier: Modellierungsprozesse) und das entwickelte Wissensprodukt (hier: Modell) von verschiedenen epistemischen Überlegungen abhängen. In diesen Überlegungen sollte die Art der Antwort, die das Modell in Bezug auf das zu lösende Problem darstellt, sowie die Generalisierbarkeit, Begründbarkeit und Zielgruppe des Modells bedacht werden (Berland et al. 2016). Bennett et al. (2020) zeigten zudem bei der Untersuchung von Modellierungsprozessen in verschiedenen Kontexten, dass SchülerInnen zum Teil aus einzelnen Worten in der Aufgabenstellung (z.B. „Population“ oder „Missbildung“) spezifische Erwartungen an die Lösung der Aufgabe ableiten und dementsprechend beim Modellieren vorgehen (z.B. aktivieren sie beim Wort „Population“ Analogien oder Erfahrungen zur Reproduktion, während bei „Missbildung" Analogien oder Erfahrungen zum Gen-zu-ProteinSchema aktiviert werden). Es liegen bislang jedoch nur vereinzelt Studien bezüglich des Einflusses epistemischer Überlegungen und spezifischer Aufgabenmerkmale in Modellierungen vor (Krell 2019; Ke und Schwarz 2020).

\section{Inwiefern treten die identifizierten Hindernissein einzelnen Phasen der Modellierungsprozesse besonders häufig auf? (Fragestellung 2)}

Hinderniskategorien, die eher explorativ und datenorientiert sind (z.B. Fehlerhafte Dokumentation, Hinweise auf unsystematischen Input, Unzufriedenheit mit den Daten), können eher in Phasen der Exploration beobachtet werden (Abb. 4). Demgegenüber treten modellierungsspezifische Hinderniskategorien auch eher in Phasen der Herstellung auf (z. B. Mangelnde Zeichenfähigkeit, Nichterkennen/Ignorieren von Mängeln am Modell, Nichterkennen/ Ignorieren von fehlender Passung). Hinderniskategorie 1 (Fehlende relevante Analogie oder Erfahrung) tritt allerdings mit nahezu allen Tätigkeiten und damit über den gesamten Modellierungsprozess hinweg auf, was erneut die zentrale Rolle der Analogiebildung für Modellierungen unterstreicht (Clement 2008; Krell et al. 2019). Das Auftreten von eher explorativen und datenorientierten Hindernissen in Phasen der Exploration und modellierungsspezifischen Hindernissen in Phasen der Herstellung macht deutlich, dass die ProbandInnen in der Lage sind eine Mehrzahl der auftretenden Hindernisse während des Auftretens zu erkennen und zu verbalisieren. Das weniger häufig beobachtete Auftreten von Hindernissen, die eigentlich eng mit einer anderen Phase des Modellierens zusammenhängen, kann dagegen die retrospektive Reflexion des eigenen Modellierungsprozesses oder sogar eine metakognitive, zielgerichtete Steuerung des eigenen Modellierungspro- zesses anzeigen (vgl. Selbstregulation; Nordheimer 2019). Es wurde jedoch kein Fall beobachtet, in dem ein Hindernis antizipiert wird, das noch gar nicht aufgetreten ist, um es aktiv zu vermeiden. Es ist davon auszugehen, dass die retrospektive Reflexion der Hindernisse im eigenen Modellierungsprozesses auf eine größere Wirkung des jeweiligen Hindernisses hindeutet, der eine Verbalisierung und Fehlersuche nötig macht, da der Problemlöseprozess nachhaltig behindert ist. Für die Phase der Vorhersage wurden keine spezifischen Hindernisse beobachtet (Abb. 2). Hierfür sind mehrere Gründe denkbar: Entweder haben ProbandInnen, die in der Lage sind, Vorhersagen aus ihrem Modell abzuleiten, bereits alle aufkommenden Hindernisse überwunden, um auf naturwissenschaftlich adäquatem Niveau modellieren zu können, oder Hindernissein Bezug auf die Vorhersage äußern sich eher im Fehlen bestimmter Tätigkeiten (z.B. fehlende Hypothesenbildung; vgl. Baur 2018). In Anbetracht der bisherigen Forschungsergebnisse zur Modellkompetenz von Lehrkräften (Krell und Krüger 2016; Nielsen und Nielsen 2019; Windschitl und Thompson 2006) erscheint letzteres wahrscheinlicher; gerade da die hier beobachteten Modellierungsprozesse durchaus variabel sind (Quigley et al. 2017) und selten Tätigkeiten der Vorhersage enthalten. Allerdings muss auch beachtet werden, dass das seltene Auftreten vorhersagender Tätigkeiten (vgl. Tab. 2) die Wahrscheinlichkeit verringert, dort typischerweise auftretende Hindernisse auch tatsächlich beobachten zu können.

Auf Ebene der Tätigkeiten (Tab. 1) können darüber hinaus die Zusammenfassung und die Prüfung der Konsistenz zwischen dem Modell und den Daten als Schlüsseltätigkeiten identifiziert werden: ProbandInnen verbalisieren am häufigsten Hindernisse während der Tätigkeit $Z u$ sammenfassung, die analog zu den Ergebnissen von Sins et al. (2005) auch die häufigste Tätigkeit darstellt (Tab. 3). ProbandInnen versuchen bei der Zusammenfassung ihre Beobachtungen zu interpretieren, ohne sie in Bezug zum Modell zu setzen, wobei besonders die explorativen und datenorientierten Hinderniskategorien auftreten. Während der Tätigkeit Prüfung der Konsistenz zwischen dem Modell und den Daten treten dagegen alle identifizierten Hinderniskategorien mindestens einmal auf (Tab. 3). Die vorliegenden Ergebnisse stützen damit die Annahme, dass das Testen von Modellen einen Schlüsselaspekt zur Förderung der Modellkompetenz von Lehrkräften darstellt (Krell und Krüger 2016; Lee und Kim 2014), da während des Testens von Modellen potenziell alle identifizierten Hinderniskategorien diskutiert werden können. 


\section{Inwiefern besteht ein Zusammenhang zwischen den identifizierten Hindernissen und den umgesetzten Modellierungsprozessen? (Fragestellung 3 )}

ProbandInnen mit ähnlichen Hindernissen können sich hinsichtlich ihrer Modellierungsprozesse deutlich unterscheiden, sodass kein eindeutiger Zusammenhang zwischen den identifizierten Hindernissen und den beobachteten Modellierungsprozessen vorzuliegen scheint (Abb. 4). Dies wird insbesondere in der kontrastierenden Fallanalyse deutlich, die aufzeigt, dass James und Jasmin zwar ähnliche Hindernisse verbalisieren, deren Umgang mit den Hindernissen aber unterschiedlich ausfällt (Abb. 5). Während James beim Auftreten von Hindernissen zuerst sein Modell verändert, konzentriert sich Jasmin auf die weitere Exploration der Blackbox, in der Hoffnung, bessere Daten zu generieren. Bei der absichtlichen Variation des Inputs geht sie jedoch eher unsystematisch vor, und möchte, wie viele der ProbandInnen, einen bestimmten Output erzielen. Dieses Vorgehen weist Parallelen zum model-fitting auf, wobei allerdings nicht das Modell, sondern die Inputmenge schrittweise variiert wird (vgl. Hogan und Thomas 2001; Sins et al. 2005). Jasmin ist in dieser Situation zwar in der Lage, das eigene Modell zu reflektieren (vgl. Windschitl und Thompson 2006) und Hindernisse zu erkennen, schafft es aber nicht, alternative Handlungen auszuführen. James dagegen nutzt sein Modell, um Hindernisse zu überwinden, indem er wiederholt Hypothesen von seinem Modell ableitet und testet. Ähnliche Muster konnten auch bei weiteren, hier nicht näher dargestellten ProbandInnen beobachtet werden. Durch das prinzipielle Auftreten ähnlicher Hinderniskategorien bei ProbandInnen, die unterschiedliche Modellierungsprozesse umsetzen, ist somit davon auszugehen, dass das Auftreten unterschiedlicher Hindernisse die Modellierung im Sinne der naturwissenschaftlichen Erkenntnisgewinnung nicht verhindert - vielmehr erscheint der Umgang mit auftretenden Hindernissen während des Modellierungsprozesses zentral für das erfolgreiche Problemlösen (Funke 2011; Mayer 2007). Hierbei sollten eine systematische Exploration und die Entwicklung kreativer Ideen and Analogien, welche das Potenzial haben, diese Beobachtungen zu erklären, als sich ergänzende Voraussetzungen gesehen werden (Krell et al. 2019).

\section{Limitationen}

Die Blackbox wurde in der vorliegenden Studie einem authentischen naturwissenschaftlichen Kontext vorgezogen, um die inhaltliche Komplexität des Problems zu reduzieren. Hierdurch ist für die Untersuchung der Blackbox kein ausgeprägtes (naturwissenschaftliches) Vorwissen notwendig und es kann auf den Problemlöseprozess fokussiert werden (,könnenszentriertes Problemlösen“; Friege und Lind
2003). Zusätzlich konnte kein systematischer Einfluss der im Samplingprozess berücksichtigten Kompetenzen des wissenschaftlichen Denkens und allgemeinen kognitiven Fähigkeiten der ProbandInnen auf die Modellierungsprozesse und beobachteten Hindernisse festgestellt werden (Göhner und Krell in Druck). Dies deckt sich mit Beobachtungen anderer Studien zu Problemlöseprozessen (vgl. Mayer 2007). Für die Modellierung der hier verwendeten Blackbox kann allerdings physikalisches Wissen hilfreich sein (z.B. im Bereich Hydraulik). Hierdurch kann eine Übertragbarkeit der vorliegenden Ergebnisse auf die Modellierung authentischer naturwissenschaftlicher Probleme limitiert sein, da wie bereits dargestellt, inhaltliches Wissen einen wichtigen Faktor für die Umsetzung von Modellierungsprozessen darstellt (Ruppert et al. 2019; Hinderniskategorie 1). Zudem können trotz der Aufforderung zum lauten Denken Teile der Denkprozesse von der Verbalisierung der ProbandInnen ausgeschlossen sein. Dabei bedingt die Beschränkung auf direkte Verbalisierungen (concurrent thinking aloud) und die beobachtbaren Handlungen auch, dass zum Beispiel mentale Modelle der ProbandInnen nicht berücksichtigt werden konnten. So wurden nur die an der Tafel gezeichneten Modelle auch als Modelle gewertet, während reine Verbalisierungen als Analogiebildung (Tab. 1) interpretiert wurden. Schließlich könnte das seltene Auftreten vorhersagender Tätigkeiten die Wahrscheinlichkeit verringert haben, entsprechende Hindernisse beobachten zu können. Eine Erweiterung der Stichprobe könnte die hierdurch angedeutete Problematik einer mangelnden Sättigung prüfen.

\section{Implikationen}

Im Rahmen der Kompetenzmodellierung und -erfassung können die identifizierten Hindernisse langfristig zur Unterscheidung von Problemtypen des Modellierens beitragen (Gut et al. 2014; Hild 2020), indem Modellierprobleme zum Beispiel auf der Basis typischer Hindernisprofile unterschieden werden. Gut et al. (2014) nutzen den Problemtypenansatz beispielsweise, um die Vielfalt experimenteller Aktivitäten in vier Problemtypen einzuteilen und für jeden Problemtyp eine spezifische Kompetenzprogression zu modellieren. Dies erlaubt die problemtypenbasierte Entwicklung von Aufgaben, die zu Messinstrumenten gebündelt werden können (z. B. Hild 2020). In Bezug auf Modellkompetenz könnte die Identifikation der hier beschriebenen Hindernisse in verschiedenen Modellierungsaufgaben damit zur Differenzierung vergleichbarer Problemtypen beitragen.

In Interventionen können die identifizierten und beschriebenen Hinderniskategorien genutzt werden, um angemessene Hilfestellungen und Impulse zu planen, die Lehrkräften bei Hindernissen im Modellierungsprozess 
spontan präsentiert werden, sodass eine nachhaltige Behinderung des Modellierungsprozesses vermieden wird (Baur 2018; Sung und Oh 2018). So sind beispielsweise bezüglich der explorativen Hinderniskategorien (z. B. Fehlerhafte Dokumentation, Hinweise auf unsystematischen Input, Unzufriedenheit mit den Daten) Hilfestellungen in Form von externen, gesicherten Datenquellen denkbar, während beim Auftreten von Hinderniskategorie 1 (fehlende relevante Analogie oder Erfahrung) eine Auswahl von Beispielmechanismen oder zu überprüfenden Modellen vorgelegt werden könnte. Im hier präsentierten Setting könnte bei Auftreten von Hinderniskategorie 8 (Zustand des Systems unklar) der aktuelle Inhalt der Blackbox vom Versuchsleiter mitgeteilt werden, ohne darüber hinaus Hinweise zum inneren Mechanismus der Blackbox zu geben.

\section{Fazit}

Die vorliegenden Ergebnisse können einen Ausgangspunkt für die Entwicklung problemtypenbasierter Kompetenzmodellierungen darstellen und so beispielsweise für die weitere Entwicklung von Diagnoseinstrumenten verwendet werden (Baur 2018; Gut et al. 2014). Gleichzeitig können mit Rücksicht auf die identifizierten Hindernisse gezielte und explizite Förderangebote konstruiert werden, die Modelle als Werkzeuge zur Erkenntnisgewinnung wahrnehmbar und die Praxis des wissenschaftlichen Modellierens auch im schulischen Kontext umsetzbar machen (Clement 2000; Lehrer und Schauble 2006). Weitere Forschung ist nötig, um die Generalisierbarkeit der gezeigten Hindernisse sicherzustellen und den Einfluss epistemischer Überlegungen und spezifischer Aufgabenmerkmale auf das Modellieren systematisch zu untersuchen.

\section{Anhang}

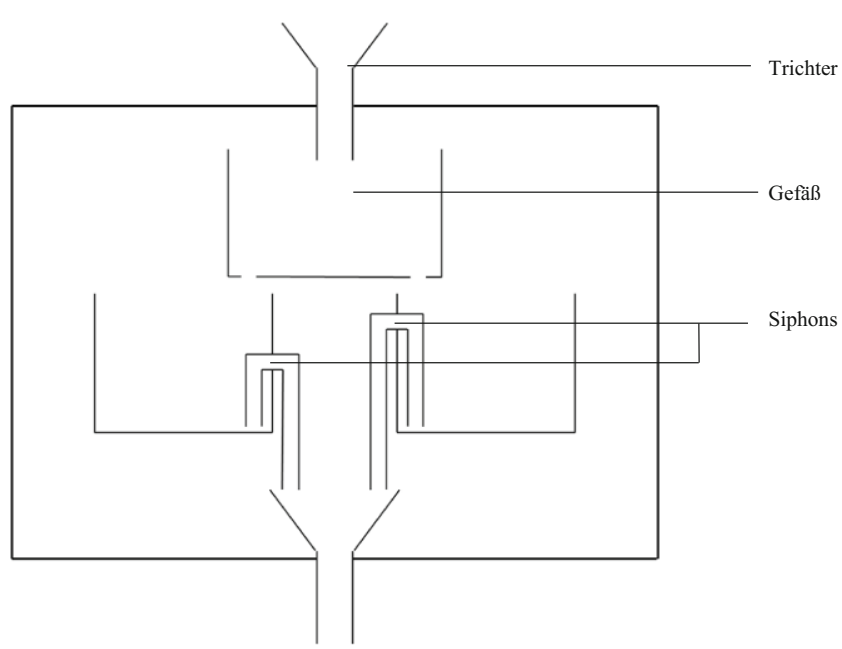

Abb. 6 Darstellung des inneren Mechanismus der Black. Wasser wird durch einen Trichter in ein erstes Gefäß geleitet, das dieses gleichmäBig auf zwei weitere Gefäße verteilt. Wird ein bestimmter Wasserstand in einem der beiden unteren Gefäße erreicht entleert sich das jeweilige Gefäß vollständig durch den Siphon. Da die beiden Siphons in unterschiedlichen Höhen angebracht sind, unterscheidet sich der eine Entleerung auslösende Wasserstand bei beiden Gefäßen (links $350 \mathrm{ml}$, rechts $550 \mathrm{ml}$ ) 
Tab. 5 Übersicht über die teilnehmenden Lehramtsstudierenden, alphabetisch geordnet nach Pseudonym

\begin{tabular}{|c|c|c|c|c|c|}
\hline Pseudonym & Alter & Studiengang & Studienfach 1 & Studienfach 2 & Untersuchungszeit \\
\hline Achilles & 25 & Bachelor & Biologie & Politik & $01: 32: 02$ \\
\hline Alexandra & 20 & Bachelor & Ernährungswissenschaften & Biologie & $01: 38: 26$ \\
\hline Alice & 22 & Bachelor & Biologie & Physik & $01: 38: 22$ \\
\hline Amy & 21 & Bachelor & Ernährungswissenschaften & Biologie & $01: 22: 13$ \\
\hline Angelina & 21 & Bachelor & Biologie & Mathematik & 01:01:17 \\
\hline Ben & 19 & Bachelor & Biologie & Politik & $01: 47: 20$ \\
\hline Boris & 19 & Bachelor & Biologie & Geschichte & 00:11:06 \\
\hline Carlo & 33 & Master & Biologie & Englisch & $01: 15: 29$ \\
\hline Celine & 17 & Bachelor & Ernährungswissenschaften & Biologie & $00: 50: 52$ \\
\hline Claire & 21 & Bachelor & Biologie & Deutsch & $01: 32: 42$ \\
\hline Claudia & 30 & Bachelor & Biologie & Politik & $00: 33: 30$ \\
\hline Cynthia & 29 & Master & Sozialkunde & Biologie & $01: 40: 43$ \\
\hline Daphne & 18 & Bachelor & Biologie & Spanisch. & $00: 50: 39$ \\
\hline Donna & 44 & Bachelor & Biologie & Philosophie & $01: 23: 46$ \\
\hline Floyd & 24 & Master & Biologie & Informatik & $00: 33: 55$ \\
\hline Frida & 29 & Master & Kunst & Biologie & $01: 49: 12$ \\
\hline Iris & 28 & Bachelor & Biologie & Mathematik & $01: 25: 16$ \\
\hline James & 39 & Bachelor & Agrarwissenschaften & Biologie & $00: 26: 37$ \\
\hline Jasmin & 25 & Master & Biologie & Geschichte & $01: 28: 25$ \\
\hline Jenny & 21 & Bachelor & Deutsch & Biologie & 01:52:03 \\
\hline Jim & 24 & Master & Biologie & Mathematik & 01:04:14 \\
\hline Jonathan & 25 & Bachelor & Biologie & Geschichte & $00: 50: 13$ \\
\hline Kara & 25 & Master & Biologie & Mathematik & 00:08:07 \\
\hline Lana & 23 & Bachelor & Englisch & Biologie & $00: 48: 33$ \\
\hline Lauren & 19 & Bachelor & Deutsche Philologie & Biologie & $01: 16: 53$ \\
\hline Martin & 33 & Master & Biologie & Chemie & $01: 52: 22$ \\
\hline May & 27 & Master & Biologie & Mathematik & $01: 27: 16$ \\
\hline Misty & 24 & Master & Biologie & Mathematik & $01: 25: 20$ \\
\hline Natalie & 21 & Bachelor & Biologie & Geschichte & $01: 23: 55$ \\
\hline Raphael & 28 & Bachelor & Biologie & Chemie & 01:05:58 \\
\hline Rocco & 24 & Master & Grundschulpädagogik & NAWI & $00: 29: 28$ \\
\hline Ryan & 30 & Master & Englisch & Biologie & 01:05:03 \\
\hline Sabrina & 18 & Bachelor & Biologie & Geschichte & 00:34:05 \\
\hline Selena & 24 & Master & Biologie & Deutsch & $00: 43: 56$ \\
\hline Susi & 33 & Master & Biologie & Politik & $01: 34: 54$ \\
\hline Valerie & 21 & Bachelor & Deutsch & Biologie & $00: 46: 21$ \\
\hline
\end{tabular}




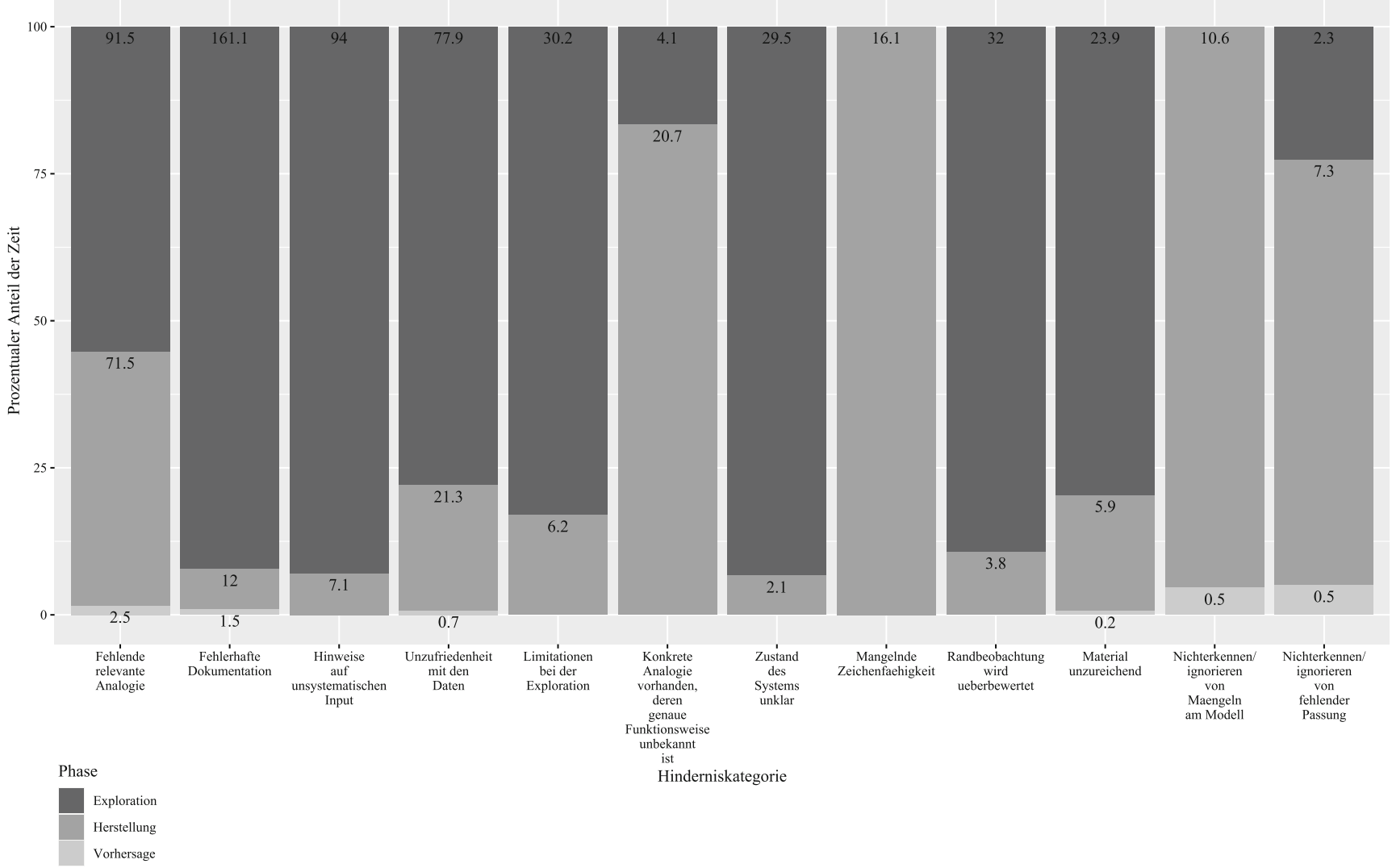

Abb. 7 Gestapeltes Säulen-Diagramm des zeitlichen Anteils aller Hindernisse unterteilt nach ihrem Auftreten in den Phasen des Modellierungsprozesses. Zur besseren Sichtbarkeit der Verhältnisse wurde der relative (prozentuale) zeitliche Anteil dargestellt. Die Säulen sind zusätzlich mit den absoluten Werten beschriftet, die die Minuten angeben, in denen das Hindernis in der jeweiligen Phase auftritt. Im Vergleich zur in Abbildung 2 dargestellten Häufigkeit der Hindernisse ergeben sich nur geringfügige Unterschiede

James

(1)
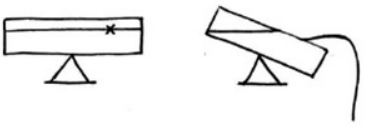

(2)

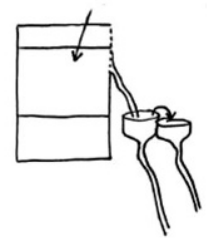

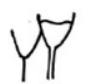
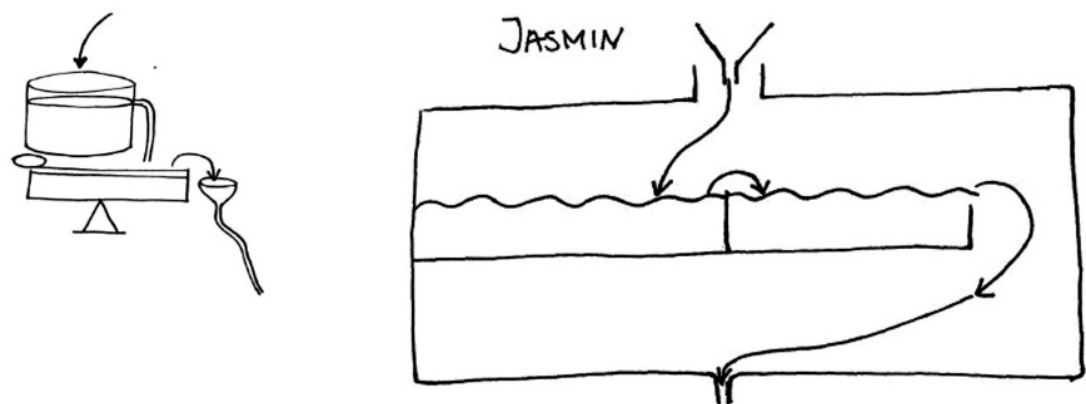

Abb. 8 Finale Modelle von James und Jasmin 
Tab. 6 Die vorgenommenen Inputs und beobachteten Outputs der beiden ProbandInnen James und Jasmin (?= Der In- oder Output wurde durch die ProbandInnen nicht verbalisiert und ist auch videografisch nicht rekonstruierbar)

\begin{tabular}{|c|c|c|c|c|c|}
\hline \multicolumn{3}{|c|}{ James } & \multicolumn{3}{|c|}{ Jasmin } \\
\hline Nr. & Input & Output & $\overline{\mathrm{Nr}}$ & Input & Output \\
\hline 1 & 400 & 0 & 1 & 400 & 0 \\
\hline 2 & 400 & 400 & 2 & 400 & 400 \\
\hline 3 & 400 & 600 & 3 & 400 & 640 \\
\hline 4 & 200 & 350 & 4 & 200 & 0 \\
\hline 5 & $?$ & $?$ & 5 & 40 & 75 \\
\hline 6 & $?$ & $?$ & 6 & 400 & $?$ \\
\hline 7 & 100 & 0 & 7 & 200 & 0 \\
\hline 8 & 100 & 0 & 8 & 100 & 0 \\
\hline 9 & 100 & 0 & 9 & 100 & $?$ \\
\hline 10 & 100 & 0 & 10 & $?$ & $?$ \\
\hline 11 & 100 & 0 & 11 & 400 & 0 \\
\hline 12 & 100 & $?$ & 12 & 400 & 400 \\
\hline \multirow[t]{13}{*}{13} & 400 & 1000 & 13 & 400 & 620 \\
\hline & & & 14 & 400 & 400 \\
\hline & & & 15 & 400 & 0 \\
\hline & & & 16 & 400 & 880 \\
\hline & & & 17 & 400 & 0 \\
\hline & & & 18 & 400 & 400 \\
\hline & & & 19 & 400 & 600 \\
\hline & & & 20 & 400 & 400 \\
\hline & & & 21 & 400 & 0 \\
\hline & & & 22 & 400 & 1000 \\
\hline & & & 23 & 1000 & 500 \\
\hline & & & 24 & 100 & $?$ \\
\hline & & & 25 & 600 & 300 \\
\hline
\end{tabular}

Danksagung Die Autoren danken der Deutschen Forschungsgemeinschaft für die finanzielle Unterstützung des Projekts TypMoL (Projektnummer 327507949).

Funding Open Access funding enabled and organized by Projekt DEAL.

Open Access Dieser Artikel wird unter der Creative Commons Namensnennung 4.0 International Lizenz veröffentlicht, welche die Nutzung, Vervielfältigung, Bearbeitung, Verbreitung und Wiedergabe in jeglichem Medium und Format erlaubt, sofern Sie den/die ursprünglichen Autor(en) und die Quelle ordnungsgemäß nennen, einen Link zur Creative Commons Lizenz beifügen und angeben, ob Änderungen vorgenommen wurden.

Die in diesem Artikel enthaltenen Bilder und sonstiges Drittmaterial unterliegen ebenfalls der genannten Creative Commons Lizenz, sofern sich aus der Abbildungslegende nichts anderes ergibt. Sofern das betreffende Material nicht unter der genannten Creative Commons Lizenz steht und die betreffende Handlung nicht nach gesetzlichen Vorschriften erlaubt ist, ist für die oben aufgeführten Weiterverwendungen des Materials die Einwilligung des jeweiligen Rechteinhabers einzuholen.

Weitere Details zur Lizenz entnehmen Sie bitte der Lizenzinformation auf http://creativecommons.org/licenses/by/4.0/deed.de.

\section{Literatur}

ACARA (2015). The Australian curriculum: science. Australian curriculum, assessment and reporting authority. http://www. australiancurriculum.edu.au/. Zugegriffen: 11. Aug. 2020.

Acher, A., Arcá, M., \& Sanmartí, N. (2007). Modeling as a teaching learning process for understanding materials: a case study in primary education. Science Education, 91(3), 398-418.

Bandura, A. (1994). Self-Efficacy. In V. S. Ramachaudran (Hrsg.), Encyclopedia of human behavior (S. 71-81). New York: Academic Press.

Baur, A. (2018). Fehler, Fehlkonzepte und spezifische Vorgehensweisen von Schülerinnen und Schülern beim Experimentieren. Zeitschrift für Didaktik der Naturwissenschaften, 24, 115-129.

BCMOE (2019). Curriculum redesign. British columbia ministry of education. https://curriculum.gov.bc.ca/rethinking-curriculum. Zugegriffen: 12. Sept. 2020.

Bell, R.L., Blair, L. M., Crawford, B. A., \& Lederman, N. G. (2003). Just do it? Impact of a science apprenticeship program on high school students' understandings of the nature of science and scientific inquiry. Journal of Research in Science Teaching, 40(5), 487-509.

Bennett, S., Gotwals, A. W., \& Long, T. M. (2020). Assessing students' approaches to modelling in undergraduate biology. International Journal of Science Education, 42(10), 1697-1714.

Berland, L.K., Schwarz, C.V., Krist, C., Kenyon, L., Lo, A.S., \& Reiser, B. J. (2016). Epistemologies in practice: making scientific practices meaningful for students. Journal of Research in Science Teaching, 53(7), 1082-1112.

Blömeke, S., Gustafsson, J.-E., \& Shavelson, R. J. (2015). Beyond dichotomies. Zeitschrift Für Psychologie, 223(1), 3-13.

Bo, W. V., Fulmer, G. W., Lee, C. K.-E., \& Chen, V.D.-T. (2018). How do secondary science teachers perceive the use of interactive simulations? The affordance in Singapore context. Journal of Science Education and Technology, 27, 550-565.

Bybee, R.W. (2002). Scientific Literacy: Mythos oder Realität? In W. Gräber, P. Nentwig, T. R. Koballa \& R. Evans (Hrsg.), Scientific literacy (S. 21-43). Wiesbaden: Springer VS.

Campbell, T., Oh, P.S., Maughn, M., Kiriazis, N., \& Zuwallack, R. (2015). A review of modeling pedagogies: pedagogical functions, discursive acts, and technology in modeling instruction. Eurasia Journal of Mathematics, Science and Technology Education, 11(1), 159-176.

Cheng, M.-F., Wu, T.-Y., \& Lin, S.-F. (2019). Investigating the relationship between views of scientific models and modeling practice. Research in Science Education. https://doi.org/10.1007/s11165019-09880-2.

Chittleborough, G., \& Treagust, D.F. (2007). The modelling ability of non-major chemistry students and their understanding of the submicroscopic level. Chemistry Education Research and Practice, 8(3), 274-292.

Chiu, M.-H., \& Lin, J.-W. (2019). Modelling competence in science education. Position Paper. Disciplinary and Interdisciplinary Science Education Research. https://doi.org/10.1186/s43031019-0012-y.

Clement, J. (1989). Learning via model construction and criticism. In J. A. Glover, R. R. Ronning \& C. R. Reynolds (Hrsg.), Handbook of Creativity (S. 341-381). Boston: Springer US.

Clement, J. (2000). Model based learning as a key research area for science education. International Journal of Science Education, 22(9), 1041-1053.

Clement, J. (2008). Creative model construction in scientists and students: the role of imagery, analogy, and mental simulation. Dordrecht: Springer.

Dörner, D. (1976). Problemlösen als Informationsverarbeitung (2. Aufl.). Stuttgart: Kohlhammer. 
Dounas-Frazer, D. R., Ríos, L., Pollard, B., Stanley, J. T., \& Lewandowski, H. (2018). Characterizing lab instructors' self-reported learning goals to inform development of an experimental modeling skills assessment. Physical Review Physics Education Research, 14(2), 20118.

Driver, R., John, L., Millar, R., \& Scott, P. (1996). Young people's images of science. Buckingham: Open University Press.

Elby, A., \& Hammer, D. (2010). Epistemological resources and framing: a cognitive framework for helping teachers interpret and respond to their students' epistemologies. In L.D. Bendixen \& F.C. Feucht (Hrsg.), Personal epistemology in the classroom: theory, research, and implications for practice (S. 409-434).

Ericsson, K.A., \& Simon, H. A. (1998). How to study thinking in everyday life: contrasting think-aloud protocols with descriptions and explanations of thinking. Mind, Culture, and Activity, 5(3), 178-186.

Fleige, J., Seegers, A., Upmeier zu Belzen, A., \& Krüger, D. (2012). Förderung von Modellkompetenz im Biologieunterricht. Der mathematische und naturwissenschaftliche Unterricht, 65(1), 19-28.

Friege, G., \& Lind, G. (2003). Allgemeine und fachspezifische Problemlösekompetenz. Zeitschrift für Didaktik der Naturwissenschaften, 9, 63-74

Funke, J. (2011). Problemlösen. In T. Betsch, J. Funke \& H. Plessner (Hrsg.), Denken - Urteilen, Entscheiden, Problemlösen (S. 136-199). Berlin, Heidelberg: Springer.

Giere, R. N., Bickle, J., \& Mauldin, R. (2006). Understanding scientific reasoning. Belmont: Thomson Wadsworth.

Gilbert, J. K., \& Justi, R. (2016). Modelling-based teaching in science education. Cham: Springer.

Godfrey-Smith, P. (2006). The strategy of model-based science. Biology and philosophy, 21(5), 725-740.

Göhner, M., \& Krell, M. (2018). Modellierungsprozesse von Lehramtsstudierenden der Biologie. Erkenntnisweg Biologiedidaktik, $17,45-63$.

Göhner, M., \& Krell, M. (2020a). Preservice science teachers' strategies in scientific reasoning: the case of modeling. Research in Science Education. https://doi.org/10.1007/s11165-020-09945-7.

Göhner, M., \& Krell, M. (2020b). Qualitative Inhaltsanalyse in naturwissenschaftsdidaktischer Forschung unter Berücksichtigung von Gütekriterien: Ein Review. Zeitschrift Für Didaktik Der Naturwissenschaften. https://doi.org/10.1007/s40573-020-00111-0.

Göhner, M., \& Krell, M. (in Druck). Analyzing the relationships between pre-service biology teachers' modeling processes, scientific reasoning competencies and general cognitive abilities. In M. Ergazaki \& K. Kampourakis (Hrsg.), Contributions from Biology Education Research. Berlin Heidelberg: Springer.

Gouvea, J., \& Passmore, C. M. (2017). "Models of" versus "Models for". Science \& Education, 26(1-2), 49-63.

Günther, S. L., Fleige, J., Upmeier zu Belzen, A., \& Krüger, D. (2017). Interventionsstudie mit angehenden Lehrkräften zur Förderung von Modellkompetenz im Unterrichtsfach Biologie. In C. Gräsel \& K. Trempler (Hrsg.), Entwicklung von Professionalität pädagogischen Personals: Interdisziplinäre Betrachtungen, Befunde und Perspektiven (S. 215-236). Wiesbaden: Springer VS.

Günther, S. L., Fleige, J., Upmeier zu Belzen, A., \& Krüger, D. (2019). Using the case method to foster preservice biology teachers' content knowledge and pedagogical content knowledge related to models and modeling. Journal of Science Teacher Education, 30(4), 321-343.

Gut, C., Metzger, S., Hild, P., \& Tardent, J. (2014). Problemtypenbasierte Modellierung und Messung experimenteller Kompetenzen von 12- bis 15-jährigen Jugendlichen. Didaktik der Physik Frühjahrstagung. https://phzh.ch/MAPortrait_Data/158541/11/Gut_ etal_2014_Problemtypbenbasierte_Modellierung.pdf. Zugegriffen: 12. Aug. 2020.

Hammann, M. (2004). Kompetenzentwicklungsmodelle: Merkmale und ihre Bedeutung - dargestellt anhand von Kompetenzen beim
Experimentieren. Der mathematische und naturwissenschaftliche Unterricht, 57(4), 196-203.

Hartmann, S., Upmeier zu Belzen, A., Krüger, D., \& Pant, H.A. (2015). Scientific reasoning in higher education: Constructing and evaluating the criterion-related validity of an assessment of preservice science teachers' competencies. Zeitschrift für Psychologie, 223(1), 47-53.

Hild, P. (2020). Problemtypenbasierte Kompetenzmodellierung beim praktisch-naturwissenschaftichen Arbeiten: Design, Validierung und Einsatz, von Aufgaben zum effektbasierten Vergleichen (Dissertation). Kiel: Christian-Albrechts-Universität.

Hodson, D. (2014). Learning science, learning about science, doing science: different goals demand different learning methods. International Journal of Science Education, 36(15), 2534-2553.

Hogan, K., \& Thomas, D. (2001). Cognitive comparisons of students' systems modeling in ecology. Journal of Science Education and Technology, 10(4), 319-345.

Justi, R., \& Gilbert, J. K. (2002). Science teachers' knowledge about and attitudes towards the use of models and modelling in learning science. International Journal of Science Education, 24(12), 1273-1292.

Justi, R., \& Gilbert, J.K. (2003). Teachers' views on the nature of models. International Journal of Science Education. 25(11), 1369-1386. https://doi.org/10.1080/0950069032000070324.

Ke, L., \& Schwarz, C. V. (2020). Supporting students' meaningful engagement in scientific modeling through epistemological messages: a case study of contrasting teaching approaches. Journal of Research in Science Teaching. https://doi.org/10.1002/tea.21662.

Khan, S. (2011). What's missing in model-based teaching. Journal of Science Teacher Education, 22(6), 535-560.

Klieme, E., Maag-Merki, K., \& Hartig, J. (2007). Kompetenzbegriff und Bedeutung von Kompetenzen im Bildungswesen. In J. Hartig \& E. Klieme (Hrsg.), Möglichkeiten und Voraussetzungen technologiebasierter Kompetenzdiagnostik (S. 5-15). Bonn \& Berlin: BMBF.

KMK (2005). Bildungsstandards im Fach Biologie für den Mittleren Schulabschluss. München \& Neuwied: Wolters Kluwer.

KMK (2019). Ländergemeinsame inhaltliche Anforderungen für die Fachwissenschaften und Fachdidaktiken in der Lehrerbildung. https://www.kmk.org/fileadmin/Dateien/veroeffentlichungen_ beschluesse/2008/2008_10_16-Fachprofile-Lehrerbildung.pdf. Zugegriffen: 12. Aug. 2020.

KMK (2020). Bildungsstandards im Fach Biologie für die Allgemeine Hochschulreife. https://www.kmk.org/fileadmin/Dateien/ veroeffentlichungen_beschluesse/2020/2020_06_18-Bildungs standardsAHR_Biologie.pdf. Zugegriffen: 16. Febr. 2021.

Knuuttila, T. (2011). Modelling and representing: An artefactual approach to model-based representation. Studies in History and Philosophy of Science Part A, 42(2), 262-271.

Koć-Januchta, M., Höffler, T., Thoma, G.-B., Prechtl, H., \& Leutner, D. (2017). Visualizers versus verbalizers: effects of cognitive style on learning with texts and pictures. Computers in Human Behavior, $68,170-179$

Krell, M. (2019). Assessment of meta-modeling knowledge: learning from triadic concepts of models in the philosophy of science. Advance online. https://doi.org/10.18452/19813.

Krell, M., \& Hergert, S. (2019). The blackbox approach: Analyzing modeling strategies. In A. Upmeier zu Belzen, D. Krüger \& J.H. van Driel (Hrsg.), Towards a competence-based view on models and modeling in science education (S. 147-160). Cham: Springer.

Krell, M., \& Krüger, D. (2013). Wie werden Modelle im Biologieunterricht eingesetzt? Ergebnisse einer Fragebogenstudie. Erkenntnisweg Biologiedidaktik, 12, 9-26.

Krell, M., \& Krüger, D. (2016). Testing models: a key aspect to promote teaching activities related to models and modelling in biology lessons? Journal of Biological Education, 50(2), 160-173. https:// doi.org/10.1080/00219266.2015.1028570. 
Krell, M., Upmeier zu Belzen, A., \& Krüger, D. (2014). Students' levels of understanding models and modelling in biology: global or aspect-dependent? Research in Science Education, 44(1), 109-132. https://doi.org/10.1007/s11165-013-9365-y.

Krell, M., Upmeier zu Belzen, A., \& Krüger, D. (2016). Modellkompetenz im Biologieunterricht. In A. Sandmann \& P. Schmiemann (Hrsg.), Biologiedidaktische Forschung: Schwerpunkte und Forschungsgegenstände (S. 83-102). Berlin: Logos.

Krell, M., Walzer, C., Hergert, S., \& Krüger, D. (2019). Development and application of a category system to describe pre-service science teachers' activities in the process of scientific modelling. Research in Science Education, 49(5), 1319-1345. https://doi. org/10.1007/s11165-017-9657-8.

Krüger, D., Hartmann, S., Nordmeier, V., \& Upmeier zu Belzen, A. (2020). Measuring scientific reasoning competencies: multiple aspects of validity. In O. Zlatkin-Troitschanskaia, H.A. Pant, M. Toepper \& C. Lautenbach (Hrsg.), Student learning in German higher education (S. 261-280). Wiesbaden: Springer VS.

Krüger, D., \& Upmeier zu Belzen, A. (2021). Kompetenzmodell der Modellierkompetenz: Die Rolle abduktiven Schließens beim Modellieren. Zeitschrift für Didaktik der Naturwissenschaften. https://doi.org/10.1007/s40573-021-00129-y

Landis, J.R., \& Koch, G.G. (1977). The measurement of observer agreement for categorical data. Biometrics, 33(1), 159-174.

Lange, D. (2013). Inhaltsanalytische Untersuchung zur Kooperation beim Bearbeiten mathematischer Problemaufgaben. Münster: Waxmann.

Lederman, N.G., \& Abd-El-Khalick, F. (2002). Avoiding de-natured science: Activities that promote understandings of the nature of science. In W. McComas (Hrsg.), The Nature of Science in Science Education. Dordrecht: Springer.

Lee, S., \& Kim, H.-B. (2014). Exploring secondary students' epistemological features depending on the evaluation levels of the group model on blood circulation. Science \& Education, 23(5), 1075-1099.

Lehrer, R., \& Schauble, L. (2006). Scientific thinking and science literacy. Handbook of child psychology, 6, 153-196.

Leisner-Bodenthin, A. (2006). Zur Entwicklung von Modellkompetenz im Physikunterricht. Zeitschrift Für Didaktik der Naturwissenschaften, 12, 91-109.

Liepmann, D., Beauducel, A., Brocke, B., \& Amthauer, R. (2007). Intelligenz-Struktur-Test 2000 R: IST 2000R. Göttingen: Hogrefe.

Louca, L.T., \& Zacharia, Z.C. (2012). Modeling-based learning in science education: cognitive, metacognitive, social, material and epistemological contributions. Educational Review, 64(4), 471-492.

Magnani, L. (1999). Model-based creative abduction. In L. Magnani, N. J. Nersessian \& P. Thagard (Hrsg.), Model-based reasoning in scientific discovery (S. 219-238). : Kluwer.

Mayer, J. (2007). Erkenntnisgewinnung als wissenschaftliches Problemlösen. In D. Krüger \& H. Vogt (Hrsg.), Theorien in der biologiedidaktischen Forschung (S. 177-186). Berlin, Heidelberg: Springer.

Meister, S., Krell, M., Göhner, M., \& Upmeier zu Belzen, A. (2020). Pre-service biology teachers' responses to first-hand anomalous data during modelling Processes. Research in Science Education. https://doi.org/10.1007/s11165-020-09929-7.

Mikelskis-Seifert, S., \& Leisner, A. (2005). Investigation of effects and stability in teaching model competence. In K. Boersma, M. Goedhart, O. de Jong \& H. Eijkelhof (Hrsg.), Research and the Quality of Science Education (S. 337-351). Dordrecht: Springer.

Nehring, A., \& Schwichow, M. (2020). Was ist Wissen, was ist Können? Deutungen des Kompetenzbegriffs und deren psychometrische Konsequenzen im Kontext von Fachwissen und Variablenkontrollstrategie. Zeitschrift für Didaktik der Naturwissenschaften. https://doi.org/10.1007/s40573-020-00113-y.

Nehring, A., Nowak, K.H., Upmeier zu Belzen, A., \& Tiemann, R. (2015). Predicting students' skills in the context of scientif- ic inquiry with cognitive, motivational, and sociodemographic variables. International Journal of Science Education, 37(9), 1343-1363.

Nersessian, N. (2002). The cognitive basis of model-based reasoning in science. In P. Carruthers, S. Stich \& M. Siegal (Hrsg.), The cognitive basis of science (S. 133-153). New York: Cambridge University Press.

Neumann, K., Härtig, H., Harms, U., \& Parchmann, I. (2017). Science teacher preparation in Germany. In J.E. Pedersen, T. Isozaki \& T. Hirano (Hrsg.), Model science teacher preparation programs: An international comparison of what works. Charlotte: Information Age.

NGSS Lead States (2013). Next generation science standards: for states, by states: appendix $\mathrm{f}$ - science and engineering practices in the ngss. In NGSS Lead States (Hrsg.), Next generation science standards: for states, by states. Washington, DC: The National Academies Press.

Nicolaou, C. T., \& Constantinou, C. P. (2014). Assessment of the modeling competence: a systematic review and synthesis of empirical research. Educational Research Review, 13, 52-73.

Nielsen, S. S., \& Nielsen, J.A. (2019). A competence-oriented approach to models and modelling in lower secondary science education: Practices and rationales among danish teachers. Research in Science Education. https://doi.org/10.1007/s11165019-09900-1.

Nordheimer, R. (2019). Volition in Modellierungsprozessen von Biologie-Lehramtsstudierenden: Entwicklung eines Kategoriensystems zur Erfassung der Selbststeuerung (unveröffentlichte Master Thesis). Freie Universität, Berlin.

Osborne, J. (2013). The 21st century challenge for science education: assessing scientific reasoning. Thinking skills and creativity, 10, 265-279.

Passmore, C. M., \& Svoboda, J. (2012). Exploring opportunities for argumentation in modeling classrooms. International Journal of Science Education, 34(10), 1535-1554.

Passmore, C. M., Gouvea, J. S., \& Giere, R. (2014). Models in science and in learning science: Focusing scientific practice on sensemaking. In M.R. Matthews (Hrsg.), International handbook of research in history, philosophy and science teaching ( $\mathrm{S}$. 1171-1202). Dordrecht: Springer.

Quigley, D., Ostwald, J., \& Sumner, T. (2017). Scientific modeling: using learning analytics to examine student practices and classroom variation. Proceedings of the Seventh International Learning Analytics \& Knowledge Conference. (S. 329-338).

Quillin, K., \& Thomas, S. (2015). Drawing-to-learn: a framework for using drawings to promote model-based reasoning in biology. CBE - Life Sciences Education. https://doi.org/10.1187/cbe.1408-0128.

Ruppert, J., Duncan, R. G., \& Chinn, C. A. (2019). Disentangling the role of domain-specific knowledge in student modeling. Research in Science Education, 49(3), 921-948.

Sandmann, A. (2014). Lautes Denken - die Analyse von Denk-, Lern- und Problemlöseprozessen. In D. Krüger, I. Parchmann \& H. Schecker (Hrsg.), Methoden in der naturwissenschaftsdidaktischen Forschung (S. 179-188). Berlin, Heidelberg: Springer.

Schauble, L., Glaser, R., Raghavan, K., \& Reiner, M. (1991). Causal models and experimentation strategies in scientific reasoning. Journal of the Learning Sciences, 1(2), 201-238.

Schreier, M. (2010). Fallauswahl. In G. Mey \& K. Mruck (Hrsg.), Handbuch Qualitative Forschung in der Psychologie (S. 238-251) Wiesbaden: VS.

Schreier, M. (2012). Qualitative content analysis in practice. London: SAGE.

Sharon, A. J., \& Baram-Tsabari, A. (2020). Can science literacy help individuals identify misinformation in everyday life? Science Education. https://doi.org/10.1002/sce.21581.

Sins, P.H., Savelsbergh, E.R., \& van Joolingen, W.R. (2005). The difficult process of scientific modelling: an analysis of novices' 
reasoning during computer-based modelling. International Journal of Science Education, 27(14), 1695-1721.

Sung, J. Y., \& Oh, P. S. (2018). Sixth grade students' content-specific competencies and challenges in learning the seasons through modeling. Research in Science Education, 48(4), 839-864.

Torres, J., \& Vasconcelos, C. (2015). Nature of science and models: comparing Portuguese prospective teachers' views. EURASIA Journal of Mathematics, Science and Technology Education, 11(6), 1473-1494.

Upmeier zu Belzen, A. (2014). Black Box: Modellierung von Prozessen naturwissenschaftlicher Erkenntnisgewinnung. In D. Ludwig, C. Weber \& O. Zauzig (Hrsg.), Das materielle Modell. Objektgeschichten aus der wissenschaftlichen Praxis (S. 99-106). Paderborn: Wilhelm Fink.

Upmeier zu Belzen, A., \& Krüger, D. (2010). Modellkompetenz im Biologieunterricht. Zeitschrift für Didaktik der Naturwissenschaften, 16, 41-57.

Vo, T., Forbes, C., Zangori, L., \& Schwarz, C. V. (2019). Longitudinal investigation of primary inservice teachers' modelling the hydrological phenomena. International Journal of Science Education, 41(18), 2788-2807.
Weinert, F. (2001). Vergleichende Leistungsmessungen an Schulen. In F. Weinert (Hrsg.), Leistungsmessungen in Schulen (S. 17-31). Weinheim, Basel: Beltz.

Werner, S., Förtsch, C., Boone, W., von Kotzebue, L., \& Neuhaus, B. J. (2019). Investigating how german biology teachers use threedimensional physical models in classroom instruction: a video study. Research in Science Education, 49(2), 437-463.

Windschitl, M., \& Thompson, J. (2006). Transcending simple forms of school science investigation: the impact of preservice instruction on teachers' understanding of model-based inquiry. American Educational Research Journal, 43(4), 783-835.

Woolley, J.S., Deal, A.M., Green, J., Hathenbruck, F., Kurtz, S. A., Park, T. K.H., et al. (2018). Undergraduate students demonstrate common false scientific reasoning strategies. Thinking skills and creativity, 27, 101-113.

Yenilmez Turkoglu, A., \& Oztekin, C. (2016). Science teacher candidates' perceptions about roles and nature of scientific models. Research in Science \& Technological Education, 34(2), 219-236. 